Irene Martorelli, Leon S. Helwerda, Jesse Kerkvliet, Sofia I. F. Gomes, Jorinde Nuytinck, Chivany R. A. van der Werff, Guus J. Ramackers, Alexander P. Gultyaev, Vincent S. F. T. Merckx and Fons J. Verbeek*

\title{
Fungal metabarcoding data integration framework for the MycoDiversity DataBase (MDDB)
}

https://doi.org/10.1515/jib-2019-0046

Received June 28, 2019; accepted April 20, 2020

\begin{abstract}
Fungi have crucial roles in ecosystems, and are important associates for many organisms. They are adapted to a wide variety of habitats, however their global distribution and diversity remains poorly documented. The exponential growth of DNA barcode information retrieved from the environment is assisting considerably the traditional ways for unraveling fungal diversity and detection. The raw DNA data in association to environmental descriptors of metabarcoding studies are made available in public sequence read archives. While this is potentially a valuable source of information for the investigation of Fungi across diverse environmental conditions, the annotation used to describe environment is heterogenous. Moreover, a uniform processing pipeline still needs to be applied to the available raw DNA data. Hence, a comprehensive framework to analyses these data in a large context is still lacking. We introduce the MycoDiversity DataBase, a database which includes public fungal metabarcoding data of environmental samples for the study of biodiversity patterns of Fungi. The framework we propose will contribute to our understanding of fungal biodiversity and aims to become a valuable source for large-scale analyses of patterns in space and time, in addition to assisting evolutionary and ecological research on Fungi.
\end{abstract}

Keywords: biogeography; data integration; DNA barcoding; environmental samples; fungal biodiversity.

\section{Introduction}

The kingdom Fungi is a major group of organisms. Fungi play essential roles in ecosystems and their ecological importance is widely studied. They are involved in the decomposition of organic carbon and contribute to the transformation of phosphorus and nitrogen compounds into crucial resources for the life of other organisms [1-3]. Fungi are found living under an extremely wide variety of environmental conditions and they have evolved diverse feeding strategies; symbiotic (i. e., intimate interactions between living partners, such as bacteria [4], plants [5, 6] and animals [7, 8]), parasitic [9] and saprotrophic (whereby fungi decompose dead organic material) [10].

\footnotetext{
*Corresponding author: Fons J. Verbeek, Leiden Institute of Advanced Computer Science (LIACS), Leiden University, Leiden, The Netherlands, E-mail: f.j.verbeek@liacs.leidenuniv.nl

Irene Martorelli: Leiden Institute of Advanced Computer Science (LIACS), Leiden University, Leiden, The Netherlands; Understanding Evolution, Naturalis Biodiversity Center, Leiden, The Netherlands

Leon S. Helwerda, Chivany R. A. van der Werff, Guus J. Ramackers and Alexander P. Gultyaev: Leiden Institute of Advanced Computer Science (LIACS), Leiden University, Leiden, The Netherlands

Jesse Kerkvliet, Sofia I. F. Gomes and Jorinde Nuytinck: Understanding Evolution, Naturalis Biodiversity Center, Leiden, The Netherlands

Vincent S. F. T. Merckx: Understanding Evolution, Naturalis Biodiversity Center, Leiden, The Netherlands; Department of Evolutionary and Population Biology, Institute for Biodiversity and Ecosystem Dynamics, University of Amsterdam, Amsterdam, The Netherlands
}

Ә Open Access. @ 2020 Irene Martorelli et al., published by De Gruyter. (๔) B Attribution 4.0 Public License. 
There are currently between 120,000 and 144,000 species [11, 12] of fungi described, but it has been estimated that the actual diversity of the group includes millions of species [13]. Yet, due to the complexity of their morphological structures, their dependencies on other organisms, and their cryptic lifestyles, much of their global distribution and diversity remains to be documented. Furthermore, the environmental factors controlling the spatial patterns of fungi are still poorly understood. Only with the advent of DNA-based molecular techniques, have researchers been able to look closely at the ecological forces that structure fungal communities. In particular, nowadays researchers make use of metabarcoding, a technique which combines high-throughput DNA sequencing methods (HTS) with a common barcode gene to identify all species present in environmental samples.

This approach has revealed new fungal species at a high rate $[14,15]$ and provides critical new insights to assessments on fungal diversity and the distribution patterns of fungal communities. For example, numerous metabarcoding studies on fungal diversity have been based entirely on DNA extracted from soil, a habitat of high fungal diversity [16, 17]. Raw DNA sequencing data from individual studies, in addition to descriptive information of the collected samples, host environments, and locations are now stored in accessible sequence read archives, for example in the National Center for Biotechnology Information (NCBI) Sequence Read Archive (SRA) [18]. These archives are therefore potential powerful sources of information for extending our knowledge of fungal distributions across multiple geographic and ecological scales. However, the metadata associated to samples is heterogeneous and information of interest is not directly accessible from the raw DNA data, hence data from individual studies are not directly comparable. These obstacles prevent large scale assessments for fungal biodiversity and distribution based on DNA data retrieved from public repositories. Therefore, here we introduce the MycoDiversity DataBase (MDDB), a curated repository of public integrated environmental samples for assisting the studies on fungal biodiversity.

In the MDDB, processed data from SRA is stored and integrated such that fungal DNA sequences are directly retrievable from a study source. MDDB facilitates navigation from individual environmental sample to a larger scale by linking the spatial components of the origins of the samples. Even though Fungi are ubiquitous in all ecosystems [19], their abundance and community structure vary across biomes and are influenced by the abiotic and biotic characteristics of their habitats. With the MDDB, a set of fungal sequences observed in refined environmental criterias can be obtained, for example fungal communities detected in very acidic soils, and researchers can explore how communities differ along particular habitat gradients. The MDDB incorporates information retrieved from public resources, including literature, SRAs and taxonomic sources used for identifying sequencing data. The integration of these data is implemented by applying three methodologies. The first one is the extensive curation applied to the annotations retrieved from the data repositories. The second is the generation of mappings for the association of data retrieved from the different data repositories. The last is the application of a uniform processing method adapted to the raw DNA data retrieved from the sequence archives. This latter method allows the incorporation of environmental traits obtained from samples and fungal DNA sequences, and thus enables to integrate data from different studies. With the inclusion of metabarcoding studies, MDDB allows to investigate the relationships of Fungi within their ecosystems, in addition to explore which are the environmental factors controlling their spatial patterns.

Our database has the objective of becoming a supportive tool for researchers to gain a better insight of the actual biodiversity of these organisms, and in addition, to facilitate the identification of the ecological fundamental features driving fungal distribution patterns over large geographic scales.

\section{Background information}

\subsection{Fungal diversity}

Traditionally, mycologists have been describing and classifying organisms belonging to this eukaryotic group by using morphological traits, mostly of sporocarps, the macroscopic reproductive structures present in some 
lineages of Fungi. This classical approach has severe limitations. For instance, many species belonging to arbuscular mycorrhizal fungi do not form visible fruiting structures [20], seasons also have an impact on sporocarp production [21] and the identification of certain groups may be restricted during their non-fruiting stages [22]. However, the recent adoption and dissemination of DNA-based molecular tools has greatly reduced the barriers to sampling and identifying fungi from fungal hyphae. The sequencing methods not only refined taxonomic relationships hypotheses which were based on morphological evidence, but also assisted the rapid identification of novel taxa [23].

The DNA barcoding technique is now a widespread sequencing approach used to identify species [23, 24], raising the actual diversity of the group to a predicted 5.1 million of species of fungi [25]. The highly variable nuclear ribosomal internal transcribed spacer (ITS) region is proposed as the primary fungal DNA 'barcode' marker for species identification [26]. HTS technologies has transformed our perspective on fungal distribution by enabling the detection of organisms directly from their host environments. Barcode sequences detected in environmental samples can be simultaneously and rapidly sequenced by using a variety of HTS platforms [27-29].

\subsection{HTS technologies on fungal diversity}

In this past decade, there has been an increasing number of environmental studies on fungal communities which relied on data obtained from HTS platforms. Fungal diversity in ecosystems have been studied over different geographical scales, such as on small-scale [30-35] and on global-scale [36-38]. Other studies focused on environmental aspects [39-41] or on the investigations of fungal-host relationships [42-45] and for observing effects of climatological gradients on biodiversity [46].

Such investigations should be carried out by applying the following criterias: the same sampling protocol for the collected samples, the equivalent HTS platform used for sequencing DNA barcode data and a uniform method for processing the raw HTS data.

\subsection{Repositories for HTS data}

The SRAs of the International Nucleotide Sequence Database Collaboration (INSDC) [47] are the European Bioinformatics Institute (EMBL-EBI) European Nucleotide Archive (ENA) [48], the NCBI SRA and the DNA DataBank of Japan Sequence Read Archive (DRA) [49]. These archives have been developed for providing the huge amount of data generated from HTS technologies in form of files containing sequence reads. As of end of April 2019, SRA contained more than 10 petabasepairs (10^16 basepairs) of open-access HTS data [50] linked to almost 200,000 published studies, in which over 4000 SRA records belong to environmental metabarcoding studies.

\subsection{Processing HTS material for fungal detection}

The raw sequencing data generated from HTS has its computational challenges for obtaining the fungal composition within an environmental sample. In order to provide species-level data for community analysis and ecological studies, the amplicon reads generated by HTS technologies need to be categorized in distinct units, which are a proxy for fungal species. Numerous bioinformatics algorithms [51-57] have been developed to cluster the raw reads based on their sequence similarity into representatives of roughly species-level, commonly referred to Operational Taxonomic Units (OTUs). The amplicon reads contain errors which are generated during the sequencing [27, 58-61]. A quality filtering step [62-64] can be applied to remove the errors from amplicon reads before the clustering procedure into OTUs. 


\subsection{Sequence based taxonomic identification}

The taxonomic annotation of OTUs relies on sequence similarity searches in reference databases. For sequence similarity searches, BLAST [65] is the most common tool. In the context of the fungal kingdom, UNITE [66] is considered as the main reference ITS database for the identification of fungi [67]. UNITE groups the ITS sequences from specimen/culture vouchers collected at several sequence similarity thresholds to obtain species-level OTUs referred as species hypotheses. All SHs (458,797 as of August 2018) [68] have a unique digital object identifier (DOI) which provides a standardized documentation of which taxa were found, and promotes unambiguous reference communication across studies [15].

\subsection{Integration of published sequence data}

Many studies rely on the use of HTS techniques for fungal assessment, but due to the different HTS platforms and the heterogeneity of the HTS data generated, the curation of these data is laborious and an uniform strategy needs to be applied. Consequently, there are very few integrated studies based on public repository data. In addition, due to the enormous species diversity of this group of organisms, many studies only focus on particular fungal groups for large-scale assessments [69].

Large-scale biogeographic signals based on HTS data are still scarce. This is mostly due to the lack of standardized approaches for extrapolating the HTS raw data. Even if guidelines do exist [14, 70], different researches apply yet different methods for denoising HTS data and decisions on OTU assignments. Not only this may affect the diversity estimate, but the direct comparison of OTUs among studies is restricted. Meiser et al., [71] were the first to exhibit a comparative metabarcoding analyses of public metabarcoding studies. Even though their approach considered only three studies deposited in SRA, the uniform denoising method applied on HTS public data, retrieved from SRA, allowed the integration of studies such to compare fungal communities on the basis of ITS sequences.

\subsection{Databases on fungal sequence data}

There have been previous attempts in building DNA-based databases taking into account fungal DNA data and associate it to information on their environment. For example, in 2010, the MaarjAM database was released [72], a repository of reference sequences belonging to the mycorrhizal fungi Glomeromycota. The database associates information about geography, habitat and climate to Glomeromycota barcode sequences. These barcodes are clustered in 'Virtual Taxa', a proxy for fungal species. However, the data deposited in MaarjAM does not include SRA data and it is only limited to published processed sequence data assigned to Glomeromycota obtained in GENBANK [73]. Several studies describing Glomeromycota have included their data in SRA (e. g., SRA study accession numbers: SRP066844, SRP087758, SRP075244, SRP067281, SRP070752). These studies reveal relationships between environmental gradients and Glomeromycota richness covering many ecosystems. MaarjAM's environmental metadata is maintained by a group of specialists who extrapolate the information from literature so that provide high quality annotation. The incorporation of new publications and sequences in the database has decreased in the past years (two publications in the years 2018-2019). This is due to the nonautomated approach of curating records and the clustering method applied which has been criticized [74].

\section{Materials and methods}

\subsection{Data acquisition}

The MDDB aims to provide a quality-controlled repository of public metabarcoding studies related to fungal diversity. The publications containing the raw HTS data submitted to sequence archives, were used for inclusion in MDDB. The workflow of the data 
acquisition methodology is summarized in Figure 1. Rather than selecting data directly from public studies, our approach was to investigate whether data could be retrievable from publications which describe studies. For the development of MDDB, our starting point was the selection of 25 publications (Appendix A) related to fungal metabarcoding studies thus by using the keywords 'ITS', 'fungi' and 'metabarcoding'. This selection all included DOIs and were all associated to HTS data.

The subsequent step involved the PubMed [75] mappings to the DOI list. We have used the NCBI standard identifier converter API [76] for retrieving the list of Pubmed identifiers of the publications. The list of Pubmed identifiers was used to obtain the PubMed records in a XML format by means of a Biopython wrapper (v.1.73) [77] on the NCBI Direct Entrez E-utilities package [78]. The records in PubMed contain formalized annotations including the abstract, authors and affiliations, and crossed-reference identifiers to other repositories. An XML parser in Python (v.2.7.15) was used to retrieve the elements and store them in a Python structure DataFrame. As from 2014, the sequence read archives of INSDC and the NCBI BioSample and BioProject databases [79] are included in the DataBankList PubMed element [80]. This element corresponds to a data source for which the accession number list of sequence data can be provided. The name of the reference repositories and their values are retrieved automatically and give rise to the PMID - SRAs mapping. For the PubMed records that do not contain this element, the complete Publication-Study mapping is achievable by retrieving the sequence read accession numbers from the publication. Our method is based on searching for regular expressions in PDF files of publications. To that end, we have defined the set of relevant regular expressions. We use PyPDF2 [81] (v.1.26.0), a PDF Python parser to find the matches to such regular expressions. The possible matches are contained in a list of sequence archive prefixes which belong to SRA, ENA and BioProject. When a match is detected, the full string is retrieved. Table 1 illustrates the list of regular expressions to search in the text of the PDF of the publications. Using this strategy, from 25 publications (88\%), we detected 22 accession numbers (Appendix A, Article Attribute). From this selection, two articles contain GENBANK identifiers which provide processed HTS data. These were not considered as they were not part of the set of regular expressions of interest and are beyond the scope of the database. Our method could not detect sequence read accession identifiers which were located in tables of the PDF files. This was the case of [71], for which a manual retrieval was applied. For the publications which reference BioProject accession numbers, the Entrez E-utilities allowed to retrieve the associated SRA study accession numbers (SRP).

The majority of the articles we have selected contained SRA as the source of sequence data (Appendix A, Study Attribute), thus this became our primary repository mapping for the related literature and SRA.

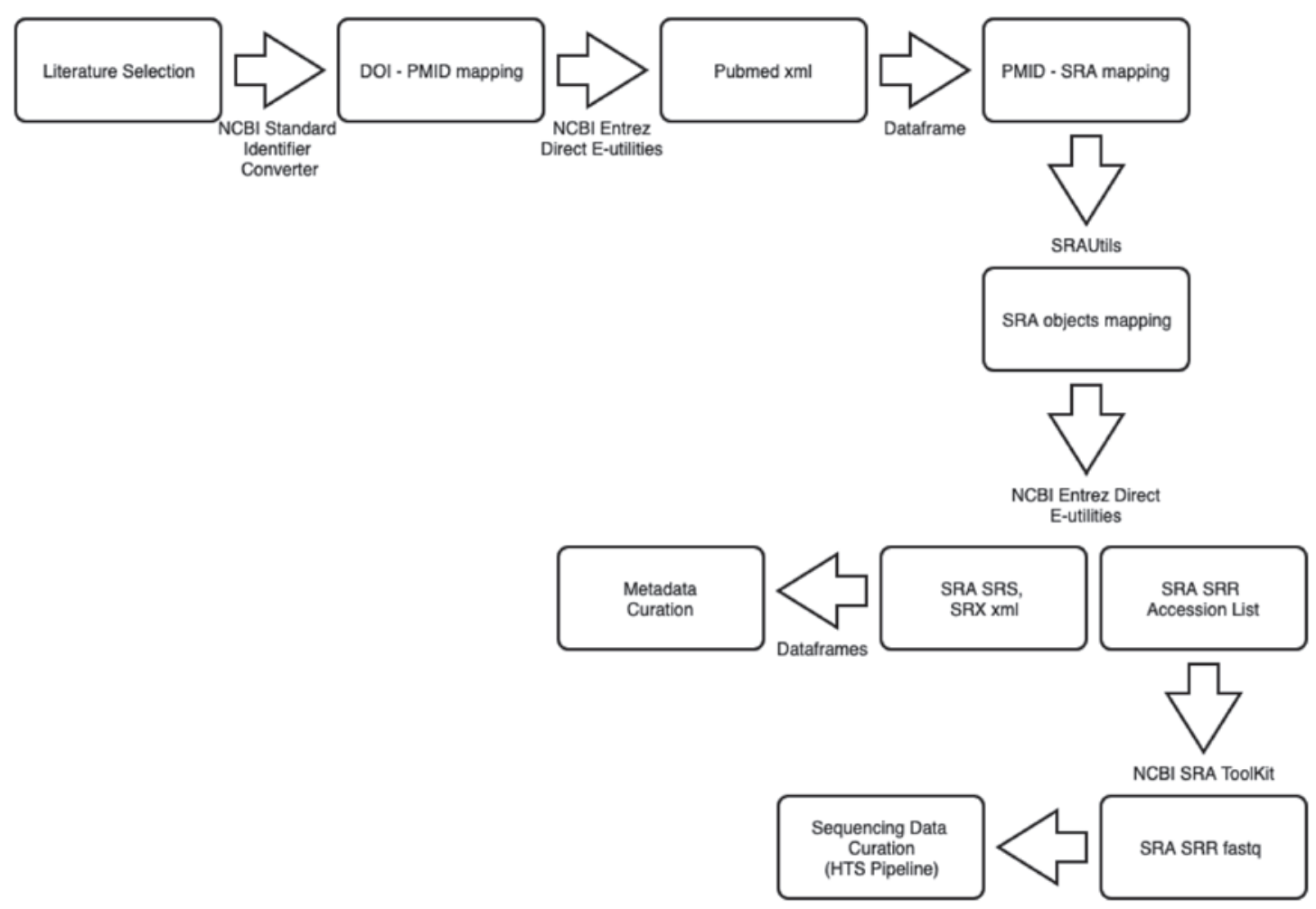

Figure 1: Data acquisition workflow for retrieving data from Pubmed and SRA repositories. Once the data is retrieved, the final step of the data acquisition involves the curation of the two data types, the metadata retrieved from PubMed and SRA (Metadata Curation), and of the raw HTS data of the SRA fastq files (Sequencing Data Curation). 
Table 1: Regular expressions used for the detection of the sequence archive prefixes in the text of publications. For each regular expression, we provide the total number of publications for which a match is detected.

\begin{tabular}{llr}
\hline $\begin{array}{l}\text { Regular } \\
\text { Expression }\end{array}$ & INSDC & $\begin{array}{r}\text { Number of } \\
\text { Publications }\end{array}$ \\
\hline SRA & DB & 5 \\
SRP & SRA & 8 \\
SRS & SRA & 2 \\
SRX & SRA & 1 \\
PRJNA & SRA & 5 \\
PRJEB & BioProject & 1 \\
ERP & BioProject & 0 \\
ERS & ENA & 0 \\
\hline
\end{tabular}

The SRA submission object domain, known simply as SRA contains 4 name space mappings, the study of research (SRP), the experiment used for sequencing (SRX), the sample of origin (SRS) and the files generated by the HTS technologies, the sequence run (SRR). In order to automatically retrieve all the object identifiers linked to an SRA object, we have used the SRAUtils (https:// bootstrappers.umassmed.edu/guides/main/), a bootstrapper for SRA Run Info CGI [82]. The metadata linked to SRP, SRX and SRS objects have been retrieved with E-search and E-fetch of the NCBI Direct E-utilities. As for the SRR objects, the raw sequence data (provided in standard flow gram format files) generated by HTS platforms, are retrieved and converted in Sanger FASTQ format by using the prefetch and fastq-dump (v.2.9.0) of the NCBI SRA Toolkit [83]. By providing in input the DOI of a publication, the data acquisition pipeline (https://github.com/naturalis/mycodiversity/tree/master/ncbi_data_acquisition) retrieves metadata associated to the SRA submission of a metabarcoding study including data linked to the NCBI BioProject and BioSample databases. The pipeline was conducted over the DOIs of the publications listed in Appendix A by using an Intel(R) Core(TM) i7-4770HQ Processor, CPU @ 2.20 GHz, 16 GB mac OS v.10.13.6 17G65 machine. As an indication, running the pipeline on one single publication which contains the PMID cross reference to SRA [44], takes 00:03:35 min for retrieving data. The data retrieved includes metadata belonging to the SRA submission mappings, 166 BioSamples, 166 experiments, mappings to the sequence run files (SRRs) and to Literature. The five files (393 KB) are all saved in a CSV format. We have compiled all the libraries in the GitHub repository (https:// github.com/naturalis/mycodiversity/blob/master/ncbi_data_acquisition/requirements.txt).

\subsection{Data curation}

3.2.1 Metadata curation and enrichment: The annotations retrieved from the resources were stored in Python DataFrames for which we have assigned categories. The 'Literature' category stores annotations acquired from the PubMed source, 'Study' contained description of the experiment conducted, the sample source, and the purpose of the research study. In 'Sequence', we have stored the run read files fetched from SRR objects.

When metadata associated to studies use standardized vocabularies, the study integration improves and the interpretation across studies is possible. Significant guidelines [84-86], are providing extensive emphasize on the application of formats, metadata standards and ontology based vocabularies for leading to knowledge integration and data reusability. Researchers who share data are aware of the importance of following standard procedures [85-89] but assigning controlled term vocabularies to the data submitted is still lacking [90]. Though SRA to be submitted requires recommended formats and standards for metadata fields, we have seen inconsistency in the format and quality in the selected studies. Because of this data heterogeneity, we have extended the annotation by the use of standard formats and terms for allowing the increase of study comparisons. The date values contained in 'Literature' and 'Study' categories have been converted to a standard format following the International Organization for Standardization protocol. The motivation of Bernstein et al., [91] to develop MetaSRA, relied on the lack of opportunity to perform large-scale analyses due to the diversity of samples in SRA and the poor structure of the metadata associated with each sample. Though their work has focused on human samples and mapping annotations to terms in biomedical ontologies, their resource shows the great impact on the use of controlled vocabularies and ontologies for the investigations across diverse conditions present in SRA metadata. For our research, it is important to curate the information related to the collected site in order to perform largescale analyses. We have observed that although controlled vocabularies are supported for the annotation of geographic location site of a sample, and it is highly recommended to provide names from the INSDC dictionary [79], yet the free text insertion is still permitted. We have used Geocoder [92] (v.1.38.1.), a geocoding Python library used for mapping the country terms to standard GeoNames [93] identifiers. The GeoNames API (http://www.geonames.org/export/web-services.html) allowed us to retrieve parent names of the list of GeoNames countries, and these were used to assign a new term 'continent' that was linked to the country name. Knowledge of the exact position on the globe where a sample is been collected is a powerful approach to fungal distribution investigation. Many studies deposited in SRA archives provide the geographical coordinates of the sample site collected; however 
these are in different formats (e. g., degrees or decimal). For allowing the integration of studies by using the coordinate location field in SRA, we have converted all coordinate values to decimal format and split this field into two distinct attributes: latitude and longitude. For including samples that do not provide coordinate values, we have used their GeoNames country identifiers for retrieving the country centroids values and assigned them to the country terms. The Google Maps javascript API (https://developers. google.com/maps/documentation/javascript/reference/) assisted in the correction of coordinate values that did not correspond to the exact spatial location displayed on a world map. This method checks and replaces the incorrect coordinate values with the GeoNames coordinate values provided in GeoNames.

Descriptions of the habitat where the sample has been collected are important for inferring their contributions on a spatial scale diversity. The environmental context, known as biome is one of the most important feature for describing the ecosystem of a sample. It is recommended to assign Environment Ontology (ENVO) [94] terms to the attributes describing the environment. We have used the EXTRACT 2.0 tool [95] for the recognition of the term provided in the SRS metadata and for the retrieval of the corresponding ENVO identifiers. All original sample metadata values are kept as linked reference to data provenance and the values which are curated are used as extended annotation.

3.2.2 HTS data curation and annotation: Because of the heterogeneity and diversity of available pipelines, we have developed a stand-alone pipeline for the generation of OTUs which encounters the diversity of the amplicon data generated by the different HTS platforms. The Processing for Fungal ITS Sequences pipeline (PROFUNGIS) (https://github.com/naturalis/mycodiversity/tree/ master/PROFUNGIS) is a pipeline developed for downloading SRA reads (SRR) and for the generation of Zero-radius OTUs (ZOTUs). For historical and technical reasons, OTUs are typically constructed using a clustering threshold similarity of 97\% [96], while for ZOTUs, a 100\% identity threshold is applied [97]. According to [98], ZOTUs have the advantage of being directly comparable between datasets without the use of reclustering. The application of ZOTUs have shown improvements in reusability and reproducibility, and according to [99, 100], they should replace the $97 \%$ OTUs as the standard unit of marker-gene analysis. The pipeline includes the UNOISE (v.3) algorithm [64], a standard algorithm in the mycological and ecological community for performing error correction on amplicon reads. The PROFUNGIS pipeline is executable by providing a set of input parameters. The mandatory arguments include: 1) forward and reverse primers used, 2) the type of ITS subunit marker sequenced 3) the platform and 4) a single SRR accession or set of SRR accessions of SRA. Following the filtering recommended steps of [101], optional parameters are also included: the minimal length overlap (minOverlap) for the merging of the Illumina read sets, the estimated error (maxEE), and the minimal filtering length for trimming. The HTS platforms which PROFUNGIS accepts are Illumina (MiSeq and HiSeq 2000 instruments), 454 (GS Junior, GS FLX Titanium and GS FLX + instruments) and Ion Torrent (PGM). In the case of Illumina platform, PROFUNGIS will include the merging step for creating consensus sequences from the paired-end reads. For the creation of the ZOTUs set, we have included the 454 GS FLX Titanium platform sequence run files belonging to the SRA Study SRP043706. This included $>3$ million raw reads $(3.11 \mathrm{~Gb}$ ). For the mandatory parameter values, we have provided the following: 1) forward and reverse primers retrieved from the design experimental metadata (SRX642180-SRX642691), 2) ITS2 marker as stated in [36], 3) platform 454 GS, retrieved from (SRX642180-SRX642691) and 4) accession list containing the sequence run accession numbers (SRR1502225SRR1502736). The default values were kept for the optional parameters, $\operatorname{maxEE}=1.0$, minOverlap $=60 \mathrm{bp}$, and minimal filtering length $=250$ for both ITS1 and ITS2 subregions of ITS. These values are recommended and are based on experimental tests during the development of PROFUNGIS and on previous studies [101], [64]. UNITE FASTA (v.7.2) release (http://doi.org/10.15156/BIO/587475) was used as a contamination filter and for the taxonomic assignment of the ZOTUs generated from each SRR file. The contamination filter uses BLAST to query the ITS ZOTUs against the UNITE database. This approach allows to remove putative ZOTUs that are not similar to known reference ITS sequences as well as to filter ZOTUs which are not associated to fungi, by using a threshold value of $70 \%$ similarity. The PROFUNGIS pipeline can run individually, but also sequentially to the data acquisition pipeline. As an indication, for a subset of 100 SRR accession numbers belonging to the SRP043706 study [36], to be converted into 629,304 (645,8 MB) FASTQ format sequences and processed to 47,529 (12,5 MB) ZOTU FASTA format sequences, PROFUNGIS takes 00:37:33 min. PROFUNGIS runs were performed by using an Intel(R) Core(TM) i7-4770HQ Processor, CPU @ 2.20 GHz, 16 GB mac OS v.10.13.6; and the pipeline can be used on other OS (https://github.com/naturalis/mycodiversity/blob/master/PROFUNGIS/ Dockerfile). We have compiled the libraries in the repository (https:/github.com/naturalis/mycodiversity/blob/master/ PROFUNGIS/requirements.txt).

\section{Implementation}

\subsection{Database design and implementation}

The MDDB is designed for the integration of metadata belonging to metabarcoding studies to processed sequence data obtained from the linked HTS data. Curated extended annotations have been linked to the original annotations for enabling the interoperability among metabarcoding studies which have been 
described in literature. The logical design of the MDDB has followed the recommended procedure of a conceptual scheme from the entity-relationship (ER) model [102]. The data has been organized and is distributed into the main conceptual components: Study, Sequence, Taxonomy, Literature and Location (Figure 2).

The concepts and information belonging to the components Literature and Study derived from data obtained from NCBI including PubMed, SRA and the BioProject and BioSample databases. Data belonging to the component Sequence is affiliated to information obtained from processed HTS data. The Taxonomy and Location components are reference repositories which further enrich the conceptual scheme by labeling the sequencing data of the Sequence component and assisting the Study and Literature components in the context of geography.

The table structure is depicted in the UML diagram of Figure 3. For the entity tables, unique internal keys, i. e., primary keys, are auto-generated. The provenance mappings of aforementioned literature and of SRA have enabled a creation of relationships within the database. The values contained in the entities Article, Journal, Author and Affiliation of the component Literature, originated from the elements automatically retrieved from PubMed. The 'Source' relation contains the mapping of studies as referred in literature and it connects the components Literature to Study. Study contains data retrieved from SRA and it concerns the relationships amongst the SRA objects.

Each sample collected for research purposes is provided in SRA with an SRS accession and it belongs to an individual study, while a study may include several samples (cf. Study.includes in Figure 3). We have observed that SRA studies may not provide sample objects, but HTS data needs to be obtained by a material (i. e., soil). Therefore, as an MDDB constraint, Sample is always defined. The relation 'Experiment' thus provides the experimental procedure conducted to a given sample and is the source for connecting the sample composition (i. e., soil) to raw data sequenced produced by an experimental strategy. We store the provenance of the run sequence files because different approaches for processing raw sequences influence the diversity estimates [71]. The PROFUNGIS pipeline has been developed for extracting biological significant sequence information from the SRA SRR files in a uniform manner. The 'Process' relation (cf. Sequence.Process in Figure 3) then stores the pipeline parameter values used for the generation of the ZOTUs. The 'ReferenceSequence' entity of
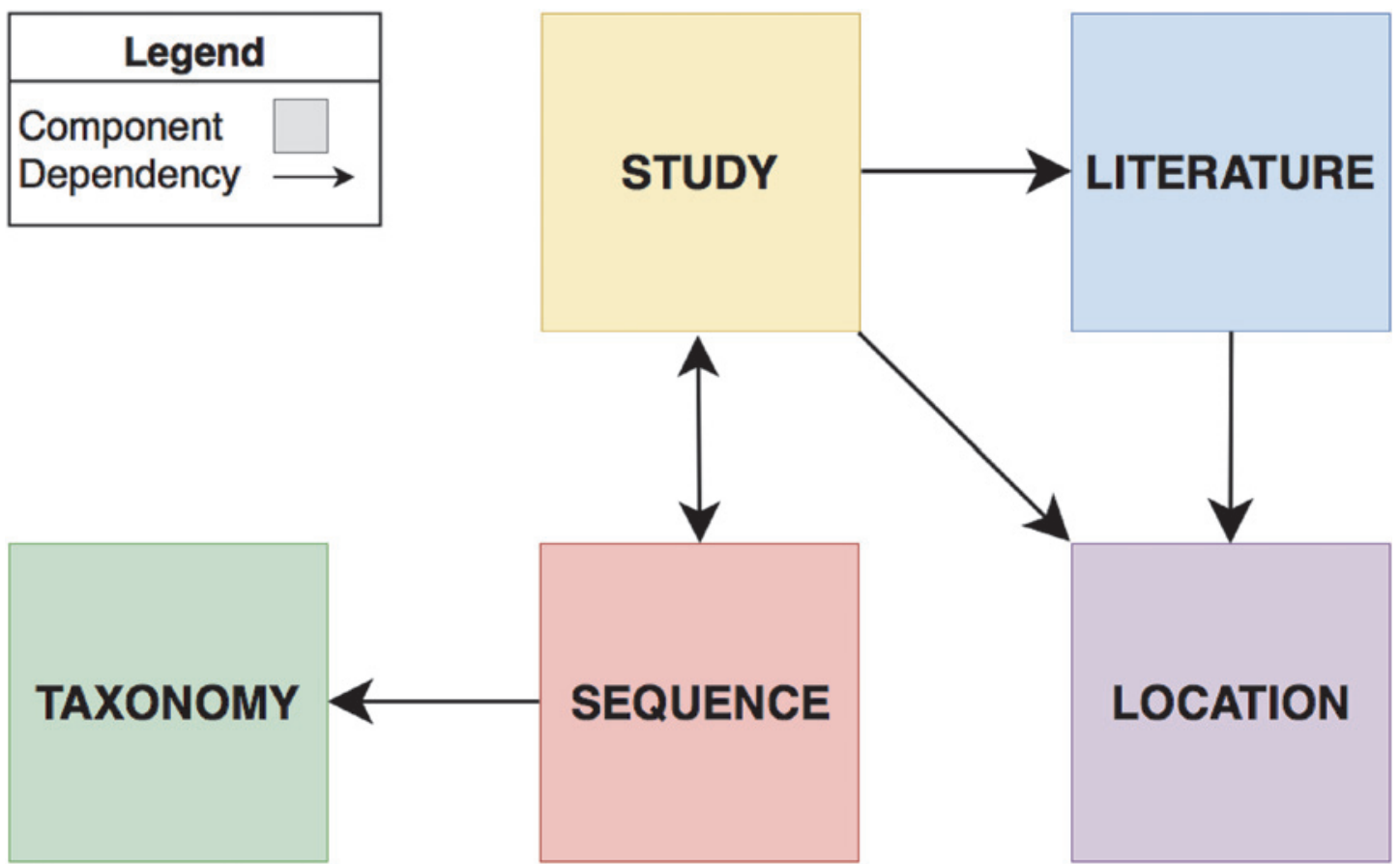

Figure 2: Components and Dependencies of the fungal metabarcoding data integration. 


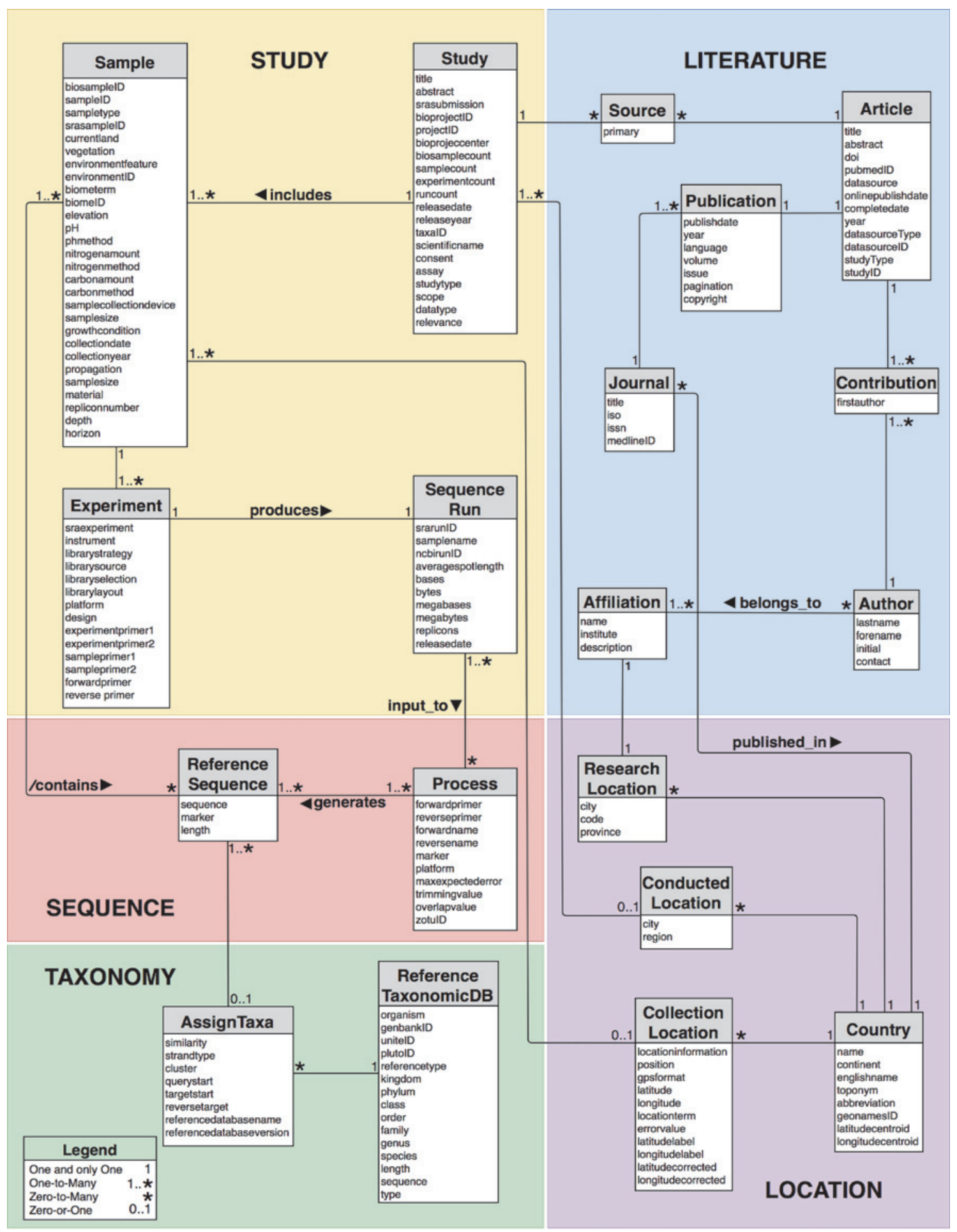

Figure 3: UML data model of the MDDB.

the Sequence component provides the unique ZOTU representatives incorporated from the processed SRR files generated in experiments. This entity also includes annotation of the barcode markers, i. e., ITS1 and ITS2 subunits, and a sequence length. Post-processing methods, such as clustering and phylogenetic reconstructions, can be applied to this set. The 'contains' mapping (cf. Sequence.contains in Figure 3) is a 
derived relationship and it guarantees the inclusion of the ZOTU reference sequences to the original sample collected and to the data provenance. This relation integrates ZOTUs into the collections of samples provided in MDDB and it is a prompt source for observing the number of shared ZOTUs among environmental conditions and to compare ZOTUs between samples. In addition, the set of ZOTU sequences of 'ReferenceSequence' have been blasted against the UNITE reference database (cf. Taxonomy.AssignTaxa in Figure 3) and the mapping provides the blast taxonomic name hit as well as the blast similarity value hit. For the Location component, the location information observed in the Study and Literature components have been related to the geographical territory names and positions by mapping to the referenced country from GeoNames information.

\subsection{Database deployment}

\subsubsection{MonetDB}

The MonetDB database-management system (DBMS) $[103,104]$ is an open source column-oriented client-server DBMS which has been particularly designed as a scalable system to be able to deal with large data volumes [105]. To that end MonetDB shows to be extremely useful for data mining applications in astronomy [106], online analytical processing applications [107], geographic information systems [108, 109] and sequence alignment processing [110]. In this latter case, the choice of MonetDB relied on previous performance tests on genome sequences [110], on response time of queries of spatio-temporal datasets [111] and on multi query optimization tests on large-scale information retrieval [106]. MonetDB delivers high performance when operating on complex and extensive queries [112] containing large amount of data entries. In addition, for our typical data we have compared the performance of SQL databases with MonetDB. Our experiments compare three DBMSs with one and the same set of test queries on the same database and on the same hardware platform. Importing the database in the different DBMSs resulted in a different size for each DBMS. These values are shown in Table 2; MonetDB uses compression [113] and therefore it is considerably smaller compared to the other two. Moreover, the results in term of execution time of all the three queries unequivocally shows that the best performance is accomplished with the MonetDB DBMS. Queries (Appendix B) were conducted on an Intel(R) Xeon(R) Processor X5355@2.66 GHz, 32 GB memory, Ubuntu OS v.16.04.4 LTS server. For all experiments, query tests were based on MonetDB version 11.27.13, MySQL version 5.7.21 and SQLite version 3.11.0.

\subsubsection{Table filling}

The values of the tables of the database schema were transposed to table templates by using the SQL-Python module (v.1.2.5.). Subsequently, the populated tables are put in MonetDB using the MonetDB MAPI library (v.1.4.0). Currently version 11.27 .13 of MonetDB is used.

Table 2: The results corresponding to the Query Tests conducted on different DBMSs. We have performed five runs for each query and taken the mean and standard deviation (SD) value. The results are displayed in seconds (unit).

Query tests conducted on different DBMS

\begin{tabular}{lrrr}
\hline & SQLite & MySQL & MonetDB \\
DBMS size (MB) & 89 & 136 & 63 \\
Query & & & 0.238 \\
Q1: Mean & 0.242 & 0.493 & 0.018 \\
Q1: SD & 0.009 & 8.606 & 0.007 \\
Q2: Mean & 0.858 & 0.005 & 0.018 \\
Q2: SD & 0.005 & 0.116 & 0.004 \\
Q3: Mean & 0.918 & 0.005 & 0.015 \\
Q3: SD & 0.003 & 0.001 \\
\hline
\end{tabular}




\subsection{Database management: PROFUNGIS post-processing method}

The PROFUNGIS pipeline generates a unique set of ZOTU representatives for every individual run file (SRR) of each sample (SRS). In order to incorporate ZOTUs amongst samples and thus updating MDDB with new samples, an automatic approach has been developed (https:/github.com/naturalis/mycodiversity/tree/ master/PROFUNGIS_post_processing). A ZOTU of a sample will be included in the database if it has not been detected yet in previous samples, and a new record in MDDB will be created to include the sequence. In sequential fashion, the 'contains' relation is updated so that the mapping of the new ZOTU representative to the original sample is incorporated in the database. Contrarily, if there is a match of the ZOTU to an existing record, it will be excluded from the ZOTU representative list and only the 'contains' relationship will be updated, such to map the ZOTU to the new sample. PROFUNGIS post processing pipeline execution depends on the size of the ZOTU Reference table. In Table 3 we present an indication of performance times of our PROFUNGIS post processing method. For each update step, we consider the incorporation to the reference dataset with 100 processed files (FASTA format) containing ZOTUs generated by PROFUNGIS. Each FASTA file contains an average range of 400-500 ZOTU sequences. For update 1, the upload included the insertion of 47,529 ZOTUs in an initial empty ZOTU reference set. This generated the set of 35,981 ZOTU representatives which were stored in the distinct RefSequence set. The number of ZOTUs after the update decreased due to detection of identical sequences, that is, common ZOTUs. The contains set takes into account this aspect thus keeping track of which ZOTUs are shared among the samples of origin. The succeeding updates follow, for which another set of 100 FASTA files are introduced in the system every time. The sequential updates are shown in Table 3; the execution time (HH:MM:SS unit) for the method to detect existing ZOTUs in MDDB increases as the RefSequence table grows. All update tests were performed on an $8 \times 2.7 \mathrm{GHz}$ Intel Xeon 5150 Processor, $16 \mathrm{~GB}$, running Debian 7 cluster node.

\section{Results}

Currently MDDB contains literature metadata obtained from 25 articles published in 2010-2017 (Table 4). The articles that provide SRA submissions, contain over $37 \mathrm{~Gb}$ of raw sequence data from which we have currently processed $2.67 \mathrm{~Gb}$ for the incorporation of ZOTUs in the RefSequence table. The processed data generated over

Table 3: Post processing update steps for the incorporation of additional FASTA files containing processed ZOTU sequence. Each update includes 100 FASTA files (average 426 ZOTUs/file) obtained from 100 distinct samples of a study. The size displayed are for csv format of the Reference ZOTU and Contain datasets while the execution time is displayed in HH:MM:SS format.

\begin{tabular}{|c|c|c|c|c|c|c|}
\hline \multicolumn{7}{|c|}{ Post processing PROFUNGIS } \\
\hline \multirow[t]{2}{*}{ Run } & \multicolumn{3}{|l|}{ Input } & \multicolumn{2}{|l|}{ Output } & \multirow{2}{*}{$\begin{array}{l}\text { Execution } \\
\text { Time }\end{array}$} \\
\hline & $\begin{array}{l}\text { ZOTUs in FASTA } \\
\text { Files }\end{array}$ & $\begin{array}{l}\text { ZOTUs in } \\
\text { Reference }\end{array}$ & $\begin{array}{l}\text { Records in } \\
\text { contains }\end{array}$ & $\begin{array}{l}\text { ZOTUs in } \\
\text { Reference }\end{array}$ & $\begin{array}{l}\text { Records in } \\
\text { contains }\end{array}$ & \\
\hline \multirow[t]{2}{*}{ Update 1} & 47529 & empty & empty & 35981 & 48086 & 00:01:01 \\
\hline & $12,5 \mathrm{MB}$ & & & $9.9 \mathrm{MB}$ & $13,6 \mathrm{MB}$ & \\
\hline \multirow[t]{2}{*}{ Update 2} & 43481 & 35981 & 48086 & 73699 & 91567 & $00: 02: 05$ \\
\hline & $11,4 \mathrm{MB}$ & $9.9 \mathrm{MB}$ & $13,6 \mathrm{MB}$ & $20,2 \mathrm{MB}$ & $26 \mathrm{MB}$ & \\
\hline \multirow[t]{2}{*}{ Update 3} & 42508 & 73699 & 91567 & 110289 & 134074 & $00: 04: 36$ \\
\hline & $11,2 \mathrm{MB}$ & $20,2 \mathrm{MB}$ & $26 \mathrm{MB}$ & $30,2 \mathrm{MB}$ & $38 \mathrm{MB}$ & \\
\hline \multirow[t]{2}{*}{ Update 4} & 42374 & 110289 & 134074 & 144193 & 177005 & $00: 05: 20$ \\
\hline & $11,1 \mathrm{MB}$ & $30,2 \mathrm{MB}$ & $38 \mathrm{MB}$ & $39,5 \mathrm{MB}$ & $50,2 \mathrm{MB}$ & \\
\hline \multirow[t]{2}{*}{ Update 5} & 37535 & 144193 & 177005 & 172463 & 213984 & $00: 06: 43$ \\
\hline & $9.9 \mathrm{MB}$ & $39,5 \mathrm{MB}$ & $50,2 \mathrm{MB}$ & $47,3 \mathrm{MB}$ & $60,7 \mathrm{MB}$ & \\
\hline
\end{tabular}


100,000 ZOTU representatives as Fungi, covering 512 samples collected in 38 countries worldwide. PROFUNGIS does rely on the input of four mandatory parameters. Two of these parameters (the SRR accessions and the SRA Experiments (SRX) attribute Platform used in the experiment setup) are provided directly from the data acquisition pipeline, while the primers can be provided in the SRX Design description.

Also the marker sequenced is never found in the experimental metadata. Therefore, we have currently processed the SRR files linked to SRX records that do provide the set of primers used within the SRX Design. For obtaining the rest of set of primers necessary to process sequence data obtained from other studies, we rely on searching for this information in the PDFs of the publications. For this approach, we have created a reference primer dataset which contain the primer names, the primer sequences, name of marker and aliases for the primer names. This primer reference table contains the list of universal primers used for ITS amplicons [114] and can be further extended with the primers described in most recent publications. These reference terms will define the set of the relevant regular expressions to search. A PDF-parser such as PDFMiner [115] or PyPDF2 [81], will allow to detect the matches of the defined regular expressions in the publications. This idea will be incorporated in the MDDB platform. Set of queries have been designed to retrieve datasets of interest. In this section we present examples of the type of data we can retrieve regarding fungal biodiversity and distribution.

\subsection{Fungal biodiversity data}

The hierarchical implementation of GeoNames applied to the location fields of the samples made the incorporation of countries to a large scale (i. e., continent) possible. As an illustration (Table 5), the diversity of major fungal groups in each major global region is retrievable.

Table 4: Type and amount of data contained in MDDB.

\begin{tabular}{ll}
\hline Data type & Total \\
\hline Articles & 25 \\
SRA submissions & 21 \\
SRA Studies (SRP) & 21 \\
454 GS & 16 \\
Illumina & 3 \\
lon Torrent & 2 \\
SRA Experiments (SRX) & 4065 \\
SRA Run Files (SRR) & $4470(37.65 \mathrm{~Gb})$ \\
SRR processed & $511(2.67 \mathrm{~Gb})$ \\
Raw sequences processed & 3037390 \\
ZOTUs generated & 172463 \\
ZOTUs assigned to Fungi & 110910 \\
SRA Samples (SRS) & 3997 \\
SRA SRS curated & 512 \\
\hline
\end{tabular}

Table 5: Amount of ZOTUs assigned for each Phylum in every continental region.

\begin{tabular}{lrrrrrr}
\hline Phylum & Africa & Asia & Europe & North America & Oceania & South America \\
\hline Basidiomycota & 3940 & 10573 & 20462 & 3865 & 8404 & 4117 \\
Ascomycota & 3862 & 9499 & 15563 & 3049 & 9542 & 6783 \\
Mortierellomycota & 207 & 1244 & 5331 & 505 & 1000 & 708 \\
Unidentified & 135 & 176 & 139 & 68 & 186 & 103 \\
Mucoromycota & 104 & 423 & 2479 & 938 & 804 & 269 \\
Chytridiomycota & 32 & 91 & 173 & 62 & 13 & 30 \\
Rozellomycota & 28 & 117 & 14 & 22 & 31 & 51 \\
Glomeromycota & 12 & 13 & 48 & & 28 & 0 \\
Other & 8 & 41 & & & & 63 \\
\hline
\end{tabular}


The taxonomic classification provided in MDDB allows to group all ZOTU representatives in each high taxonomic rank (phylum). As a result, for each continent, Basidiomycota turned out to have the most ZOTU representatives (Figure 4), with the exception of Oceania and South America, were Ascomycota where more abundant in terms of diversity.

\subsection{Fungal distribution data}

The taxonomic hierarchy and the curated coordinate values of samples deposited in MDDB allow to display diversity patterns and distribution of specific taxonomic group of fungi. As an example, in Figure 5 we illustrate the distribution of the Russulaceae family within the spatial range among the tropic of Cancer and tropic of Capricorn. The map also displays the diversity of the family based on the distinct ZOTU occurrences for each plot. Queries can be further extended for obtaining set of ZOTU sequences observed in samples and display taxonomic distributions in refined environmental criterias, for example by comparing species richness among biomes.

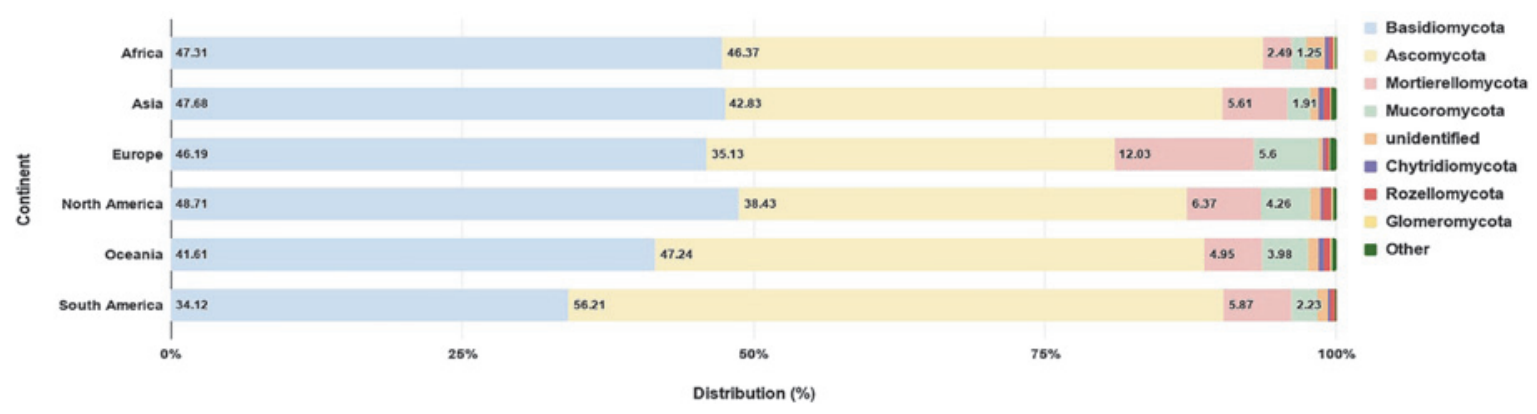

Figure 4: Major phylum ZOTU representatives for each continent.

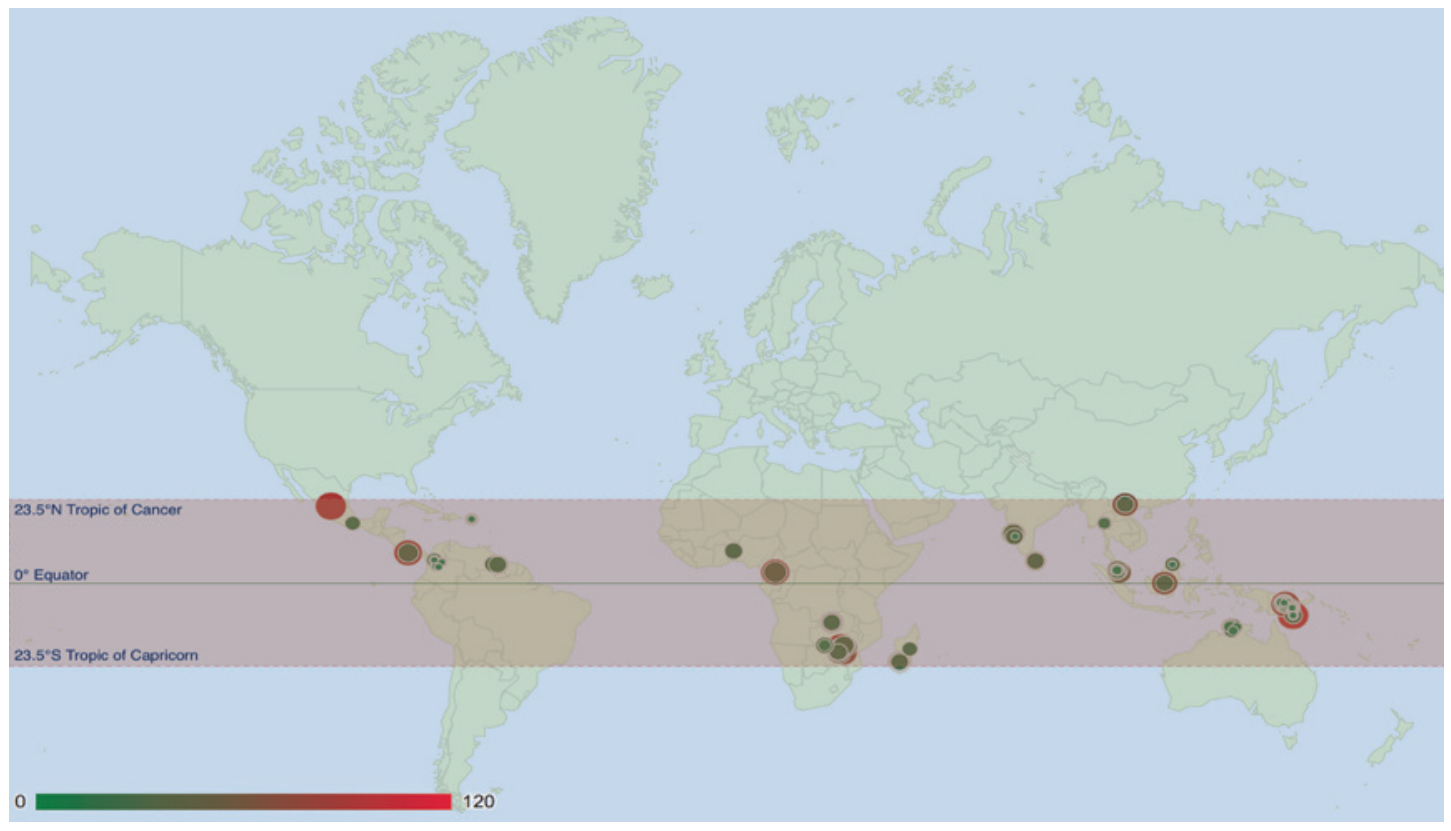

Figure 5: Diversity of ZOTUs belonging to the Russulaceae family and their distribution display in a spatial range. Map display by using Marker GeoCharts (http://developers.google.com/chart/interactive/docs/gallery/geochart). 


\subsection{Shared ZOTUs among continents}

The adaptation of a uniform pipeline to process HTS data, has allowed the incorporation of ZOTUs among samples. MDDB permits not only to observe fungal composition within a sample, but also allows to observe which global regions and environmental conditions have the most similar fungal community composition as revealed by the shared fungal sequences observed in the different locations. Table 6 shows the type of data associated with each continent and the number of ZOTUs in the current version of MDDB. The data is currently biased because most of the samples included in MDDB are from Asia, Europe and Oceania. With MDDB, we are able to retrieve the amount of ZOTUs for a given location and compare it with the rest of the globe. Europe and Asia share a comparatively high number of shared ZOTUs between the rest of the globe.

Meiser et al., [71], presented the amount of shared OTUs among studies and with a similar approach, MDDB provides the possibility to display the fungal similarities and comparisons among locations and compare a specific region composition with the rest of the globe. The pie-charts in Figure 6, illustrate the geographical distribution of the ZOTUs that occur on more than one continent. The number of samples and number of ZOTUs included in the continents Oceania and Asia are more similar to each other compared to the other continents, while the number of shared ZOTUs among Asia and Europe is considerably high compared to the shared ZOTUs between Europe and Oceania. Appendix C shows the queries constructed to generate the output results displayed in this section.

Table 6: ZOTUs representatives for each continent and common ZOTUs among continents.

\begin{tabular}{|c|c|c|c|c|c|c|c|}
\hline Amount & Globe & Africa & Asia & Europe & $\begin{array}{r}\text { North } \\
\text { America }\end{array}$ & Oceania & $\begin{array}{r}\text { South } \\
\text { America }\end{array}$ \\
\hline Number of countries & 38 & 5 & 11 & 10 & 5 & 3 & 4 \\
\hline Number of samples & 512 & 35 & 85 & 217 & 28 & 88 & 59 \\
\hline Number of ZOTUs & 172463 & 13355 & 33178 & 68566 & 11414 & 31247 & 19324 \\
\hline Number of ZOTUs shared betweeen continents & & 497 & 2192 & 2191 & 926 & 1306 & 929 \\
\hline
\end{tabular}

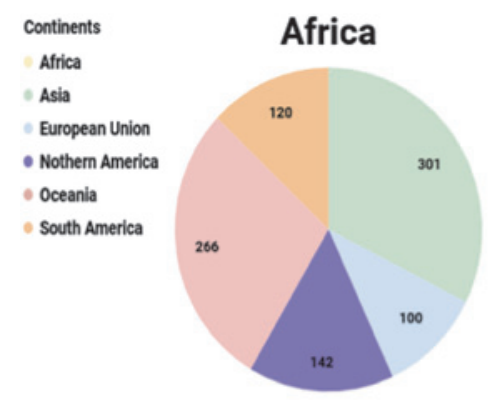

Nothern America

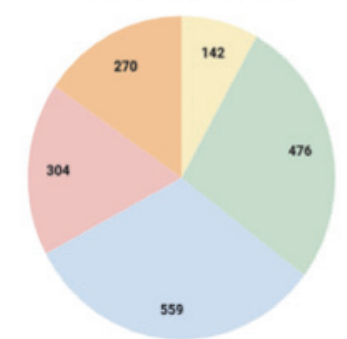

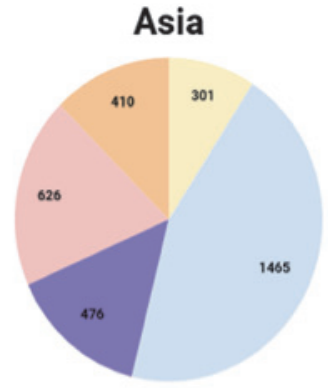

Oceania

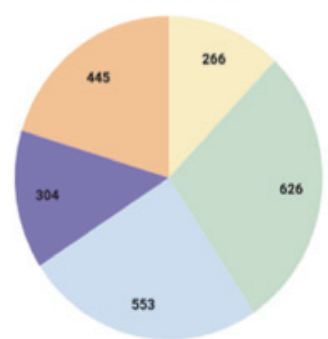

Europe

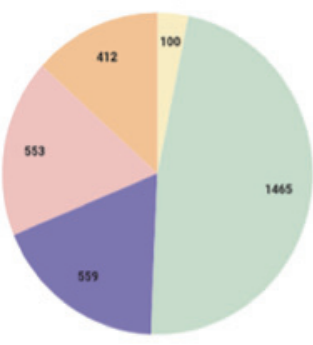

South America

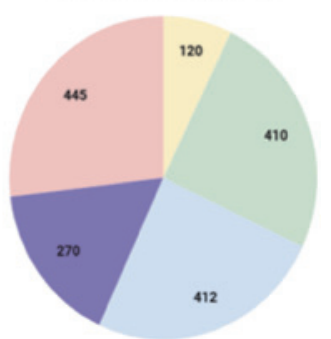

Figure 6: Overlap of ZOTUs among continents. 


\section{Discussion}

The FAIR principles [86] have been leading in the design, implementation and deployment of the MDDB. An important result of our framework, we provide reliable high quality data in a feasible manner so as to facilitate the community for fungal biodiversity discovery. Additionally, MDDB allows investigating the environmental contributions to fungal distribution and biodiversity. The consistent structure of MDDB assures maintaining the integrity of data pertaining publicly available metabarcoding studies. For our implementation, the FAIR principles for data enrichment with standard terms and methods as well as controlled vocabularies and ontologies are applied. This assures good data integration and data longevity. It is a crucial factor in the further development of MDDB.

\subsection{Data feasibility and accessibility}

Accessing data that is stored across different public sources in a feasible manner is one of the keys for leading to knowledge discoveries. The tools Entrez direct E-utilities, NCBI Run browser and Run selector [116] permit data selection and data retrieval associated with SRA. However, these utilities are only efficient when the Entrez Unique Identifiers are provided in constructing the search (for example, with using SRA study accession numbers), but the selection becomes challenging when text search is applied. Furthermore, the raw sequence data associated to SRA records is only achievable when SRR accessions numbers are used in combination with the SRA Toolkit. The packages SRAdb [117] and SRAmongo [118] facilitate the access and data retrieval from the SRA database. They also tend to aggregate data from the NCBI interlinked databases (e. g., SRA and PubMed), however data from the publication is retrievable if only the databases cross-reference is interoperable. Regarding interoperability of the NCBI resources (Appendix A, PMID and SRA Cross Reference attributes) there are inconsistencies. For example, by using the NCBI Run selector, the PMID of the associated article was not found in the namespace of Tedersoo's SRA study [36]. With our data acquisition method (Section 3.1), the SRA study is directly retrievable by using the DOI of the publication. To the best of our knowledge, MDDB is the first to provide the complete mapping of NCBI publications to SRA based on a selected list. The complete mapping has been achieved by retrieving the study identifiers from the PDFs of the publications for which this information is not contained in the associated PubMed. This relationship facilitates the selection of studies and experimental assays by using literature text terms (e. g., article title and keywords within an abstract). Additionally, it allows the direct access of publications describing mycodiversity to its sequencing data (e. g., a list of fungal species detected can be retrieved by a publication title).

\subsection{Data integration and interoperability}

The integration of environmental samples for the exploration of global fungal diversity and distribution can be achieved if metadata retrieved from public domains is formalized, curated and standardized. At the same time uniform methods must be applied to the raw HTS data. Data associated with studies is increasingly made available in public data repositories $[119,120]$ making this a valuable source for our domain. However, while doing our research, we have observed the lack of standard terms and controlled vocabularies used to describe biological samples in SRA. We showed the potential of mapping location annotations to Geonames terms, ENVO terms to the habitat annotation and apply standardization to the coordinate values (Section 3.2.1). This data enrichment offers the opportunity to increase relationships between geographical regions and fungal communities across diverse environmental conditions, thereby increasing the interoperability among studies.

We believe that extending the application of semantic mappings to submitted data will reduce heterogeneity and increase integration of studies. We want to further enrich MDDB with ENVO vegetation and materials terms (i. e., soil) and assign CheBI [121] terms to the nutrient components, such as carbon and nitrogen and to conditions, for instance to the hydrogen values used for the acidity of the soil. Furthermore, we will apply the Units Ontology [122] to control the formats of units in association to measurements, such as to the concentration and amount of compounds found in the environmental material and to the soil depth and 
amount of sample collected. Moreover, we will include the application of the ENVO ontological classification for extending the integration of studies by using biomes terms.

Finally, we emphasize the application of text mining tools for the analysis of publications, such as the use of EXTRACT to retrieve possible terms within the text. Usually in a publication there is an extensive description of the habitat. Natural language processing methods will allow the retrieval the NCBITaxon terms (i. e., plant species names) constituting the associate host organisms from which a sample has been collected. The essential parameters for processing the sequence data (i. e., primers, barcode type) can be obtained by searching for the relevant regular expressions in the PDF of the publication as proposed here; these valuable annotations will be included in MDDB in a standard format.

MDDB is the first repository which provides reliable sets of ZOTUs generated by a supportive pipeline for denoising HTS data from the widely used HTS platforms and which have been deposited in SRA. The choice of an uniform methodology allows to directly compare the generated ZOTUs and the taxonomic composition among studies. The low number of shared OTUs among the studies selected by Meiser et al., [71] has suggested high global fungal diversity and indicates that globally distributed taxa may be rare. Increasing the number of SRA samples in MDDB, will allow to identify more shared ZOTUs among studies and determine which fungal communities are highly similar among compared studies.

\subsection{Data reusability}

MDDB is a reliable system to which its enriched metadata is always associated to the provenance and thirdparty annotations by the use of URIs. It is important to emphasize at this stage that data will be made accessible and retrievable by means of a high-end interface and an API. The prototype user interface (https:// mycodiversity.liacs.nl) gives insight of the data contained in MDDB, and is leading to serve as a guide for comparative studies as well as constructing both local and global scale assessments for fungal discoveries. Data are reusable and reproducible, as for now by the use of the tools (https://github.com/naturalis/ mycodiversity) and by means of extensions which will be included in the future interfaces.

MDDB will incorporate more SRA studies and consequently include more data. The MonetDB DBMS guarantees high performance on queries against big datasets and this can auxiliate users to efficiently select and retrieve data. It is our intention to allow the navigation of public fungal metabarcoding data in a feasible, straightforward manner, such that the user will not spend unnecessary time in the curation and organization of data [123] and enhance time for fungal analysis.

\section{Final remarks}

The rationale of this paper is to illustrate our framework for the curation and integration of fungal metabarcoding data which is compiled in the MDDB database. With this dedicated database, we now can efficiently retrieve data from collections of fungal metabarcoding studies. The database can be further extended with the latest studies using all the tools that we have presented for integration and curation. The integration and curation processes guaranteed uniformity of the data in MDDB, thereby accomplishing an enhancement of availability of public domain fungal metabarcoding data. The database and the pipelines are now ready for large-scale application.

The further extension of the GUI is complementary to the integration effort. The interface is built from predefined queries. We start with relatively simple predefined queries and as the interface evolves more complex queries can be composed. This is part of the future work, some of the ideas are already implemented. The usability within the community is leading the further development of the user interface.

All considered, this database is ready to support and facilitate large-scale research in fungal biodiversity.

Conflict of interest: Authors state no conflict of interest. All authors have read the journal's Publication ethics and publication malpractice statement available at the journal's website and hereby confirm that they comply with all its parts applicable to the present scientific work. 


\section{Appendix A}

Table A.1: Selection of publications describing metabarcoding studies.

\begin{tabular}{|c|c|c|c|c|c|c|}
\hline Article & Year & PMID & Study & SRA Study Title & $\begin{array}{l}\text { PMID } \\
\text { C.R. }\end{array}$ & $\begin{array}{l}\text { SRA } \\
\text { C.R. }\end{array}$ \\
\hline $\begin{array}{l}\text { 1. Fungal biogeography. Global di- } \\
\text { versity and geography of soil fungi. } \\
\text { DOI: } 10.1126 / \text { science. } 1256688\end{array}$ & 2014 & 25430773 & SRP043706 & Global fungal diversity & $\mathrm{Y}$ & Null \\
\hline $\begin{array}{l}\text { 2. New primers to amplify the fungal } \\
\text { ITS2 region-evaluation by } 454- \\
\text { sequencing of artificial and natural } \\
\text { communities. } \\
\text { DOI: } 10.1111 / j .1574-6941.2012 \text {. } \\
01437 . x\end{array}$ & 2012 & 22738186 & SRP012868 & $\begin{array}{l}\text { Design and test new primers to be } \\
\text { used to amplify the fungal ITS } 2 \text { re- } \\
\text { gion by targeting sites in the } 5.8 \mathrm{~S} \\
\text { encoding gene }\end{array}$ & Null & Null \\
\hline $\begin{array}{l}\text { 3. Strong altitudinal partitioning in } \\
\text { the distributions of ectomycorrhizal } \\
\text { fungi along a short }(300 \mathrm{~m}) \text { elevation } \\
\text { gradient. } \\
\text { DOI: } 10.1111 / \mathrm{nph} .13315\end{array}$ & 2015 & 25655082 & SRP043982 & Root associated fungi Metagenome & $\mathrm{Y}$ & Null \\
\hline $\begin{array}{l}\text { 4. Roots and associated fungi drive } \\
\text { long-term carbon sequestration in } \\
\text { boreal forest. } \\
\text { DOI: } 10.1126 / \text { science. } 1231923\end{array}$ & 2013 & 23539604 & SRP016090 & $\begin{array}{l}\text { The Island Project - Fungal com- } \\
\text { munities in boreal forest soils }\end{array}$ & Null & Null \\
\hline $\begin{array}{l}\text { 5. ITS1 versus ITS2 as DNA meta- } \\
\text { barcodes for fungi. } \\
\text { DOI: } 10.1111 / 1755-0998.12065\end{array}$ & 2013 & 23350562 & SRP026239 & $\begin{array}{l}\text { Analysis of ITS1 and ITS2 regions for } \\
\text { barcoding fungal specimens }\end{array}$ & Null & $\mathrm{Y}$ \\
\hline $\begin{array}{l}\text { 6. Dispersal in microbes: fungi in } \\
\text { indoor air are dominated by outdoor } \\
\text { air and show dispersal limitation at } \\
\text { short distances. } \\
\text { DOI: } 10.1038 / \text { ismej. } 2013.28\end{array}$ & 2013 & 23426013 & SRP015917 & $\begin{array}{l}\text { Fungal ITS amplicons from airborne } \\
\text { household dust }\end{array}$ & Null & Null \\
\hline $\begin{array}{l}\text { 7. Quantifying microbial commu- } \\
\text { nities with } 454 \text { pyrosequencing: } \\
\text { does read abundance count? } \\
\text { DOI: } 10.1111 / \text { j.1365-294X.2010. } \\
\text { 04898.x }\end{array}$ & 2010 & 21050295 & SRP001800 & $\begin{array}{l}\text { Pyrosequencing of Global House } \\
\text { Dust }\end{array}$ & Null & $\mathrm{Y}$ \\
\hline $\begin{array}{l}\text { 8. Indoor fungal composition is } \\
\text { geographically patterned and mor- } \\
\text { ediverse } n \text { temperate zones than in } \\
\text { the tropics. } \\
\text { DOI: } 10.1073 / \text { pnas. } 1000454107\end{array}$ & 2010 & 20616017 & SRP001800 & $\begin{array}{l}\text { Pyrosequencing of Global House } \\
\text { Dust }\end{array}$ & Null & Y \\
\hline $\begin{array}{l}\text { 9. Spatio-temporal dynamics of soil } \\
\text { bacterial communities as a function } \\
\text { of Amazon forest phenology. } \\
\text { DOI: } 10.1038 / \text { s41598-018-22380-z }\end{array}$ & 2018 & 29531240 & GENBANK & Null & Null & Null \\
\hline $\begin{array}{l}\text { 10. Phylogenetic relatedness ex- } \\
\text { plains highly interconnected and }\end{array}$ & 2017 & 28207957 & SRP042134 & $\begin{array}{l}\text { Plant roots in subtropical forest } \\
\text { Metagenome }\end{array}$ & $\mathrm{Y}$ & Null \\
\hline
\end{tabular}
nested symbiotic networks of woody plants and arbuscular mycorrhizal fungi in a Chinese subtropical forest.

DOI: $10.1111 / \mathrm{mec} .14061$ 
Table A.1: (continued)

\begin{tabular}{lllllll}
\hline Article & Year & PMID & Study & SRA Study Title & $\begin{array}{l}\text { PMID } \\
\text { C.R. }\end{array}$ & $\begin{array}{l}\text { SRA } \\
\text { C.R. }\end{array}$ \\
\hline $\begin{array}{l}\text { 11. Convergence and contrast in the } \\
\text { community structure of Bacteria, }\end{array}$ & 2017 & 28402397 & SRP102378 & Hawaii Soils Raw sequence reads & Null & Null \\
\hline
\end{tabular}

Fungi and Archaea along a tropical

elevation-climate gradient.

DOI: $10.1093 /$ femsec/fix045

12. FUNGAL SYMBIONTS. Global

assessment of arbuscular mycor-

rhizal fungus diversity reveals very

low endemism.

DOI: $10.1126 /$ science.aab1161

13. Arbuscular mycorrhizal in-

teractions of mycoheterotrophic

Thismia are more specialized than in autotrophic plants.

DOI: $10.1111 / \mathrm{nph} .14249$

14. A phosphorus threshold for mycoheterotrophic plants in tropical forests.

DOI: $10.1098 / \mathrm{rspb} .2016 .2093$

15. Fungal-host diversity among mycoheterotrophic plants increases proportionally to their fungal-host overlap.

DOI: $10.1002 /$ ece 3.2974

16. Consistent responses of soil microbial communities to elevated nutrient inputs in grasslands across the globe.

DOI: $10.1073 /$ pnas.1508,382,112

17. Severe plant invasions can increase mycorrhizal fungal abun-

dance and diversity.

DOI: 10.1038 /ismej.2013.41

18. Host identity is a dominant driver of mycorrhizal fungal community composition during ecosystem development.

DOI: $10.1111 / \mathrm{nph} .13226$

19. Fungal community analysis by large-scale sequencing of environmental samples.

DOI: 10.1128/AEM.71.9.5544-

5550.2005

20. Comparison and Validation of Some ITS Primer Pairs Useful for Fungal Metabarcoding Studies DOI: 10.1371/journal.pone. 0097629
Global assessment of arbuscular

Null Null mycorrhizal fungus diversity reveals very low endemism

$201727739593 \quad$ SRP083901

Arbuscular mycorrhizal interactions

Null

Null more specialized than autotrophic plants

28148744

SRP076949

Study of fertilization, litter addition, and soil phosphorus effects on fungal and arbuscular mycorrhizal fungal communities in Panamanian tropical forest 28515898 SRP082976

Host diversity increases proportionally to host overlap among mycoheterotrophic plants

RP052716

Soil marker gene sequences across the Nutrient Network

Null

ference drives mycor throughout ecosystem development

200516151147 GENBANK Null

Null Null 
Table A.1: (continued)

\begin{tabular}{|c|c|c|c|c|c|c|}
\hline Article & Year & PMID & Study & SRA Study Title & $\begin{array}{l}\text { PMID } \\
\text { C.R. }\end{array}$ & $\begin{array}{l}\text { SRA } \\
\text { C.R. }\end{array}$ \\
\hline $\begin{array}{l}\text { 21. Lack of host specificity leads to } \\
\text { independent assortment of diptero- } \\
\text { carps and ectomycorrhizal fungi } \\
\text { across a soil fertility gradient. } \\
\text { DOI: } 10.1111 / \text { ele.12459 }\end{array}$ & 2015 & 26032408 & SRP057798 & $\begin{array}{l}\text { Root associated fungi Targeted loci } \\
\text { environmental }\end{array}$ & Null & $\mathrm{Y}$ \\
\hline $\begin{array}{l}\text { 22. Meta-analysis of deep- } \\
\text { sequenced fungal communities in- } \\
\text { dicates limited taxon sharing be- } \\
\text { tween studies and the presence of } \\
\text { biogeographic patterns. } \\
\text { DOI: } 10.1111 / \text { nph.12532 }\end{array}$ & 2017 & 24111803 & Multi & Null & Null & Null \\
\hline $\begin{array}{l}\text { 23. Sequence Depth, Not PCR } \\
\text { Replication, Improves Ecological } \\
\text { Inference from Next Generation DNA } \\
\text { Sequencing. } \\
\text { DOI: } 10.1371 \text { /journal.pone. } \\
0090234\end{array}$ & 2014 & 24587293 & SRP035367 & DOB_RepEx Targeted Locus (Loci) & Null & $\mathrm{Y}$ \\
\hline $\begin{array}{l}\text { 24. Strong linkage between plant } \\
\text { and soil fungal communities along a } \\
\text { successional coastal dune system. } \\
\text { DOI: } 10.1093 / \text { femsec/fiw156 }\end{array}$ & 2016 & 27411980 & SRP059280 & $\begin{array}{l}\text { Ectomycorrhizal fungal commu- } \\
\text { nities in a relic foredune plain }\end{array}$ & Null & Null \\
\hline $\begin{array}{l}\text { 25. Fungal endophyte communities } \\
\text { reflect environmental structuring } \\
\text { across a Hawaiian landscape. } \\
\text { DOI: } 10.1073 / \text { pnas. } 1209872109\end{array}$ & 2012 & 22837398 & SRP013695 & $\begin{array}{l}\text { Fungal ITS1 amplicon library from } \\
\text { environmental samples Targeted } \\
\text { Locus (Loci) }\end{array}$ & Null & Null \\
\hline
\end{tabular}

\section{Appendix B: Example queries used for comparing the performance in different DBMS, Section 4.2.1, Table 2}

\section{ZOTU representatives in single location}

\section{Query 1 (Q1): provide all sequence representatives (ZOTUs) from a specific sample}

\section{SELECT DISTINCT RS. Sequence_pk, RSM. Amount}

FROM SampleMetadata1 SM, RefSampleSequence RS, RefSeqSampleMapping RSM

WHERE SM. Sample_id = 'SRS651086' AND SM. Sample_pk = RSM. Sample_pk AND

RSM. Sequence_pk = RS. Sequence_pk;

530 tuples

\section{Specific ZOTUs compare to another location}

Query 2 (Q2): provide ZOTUs which are specific in one region and not found in another region of the same country. 
WHERE SM. Country_name = 'Estonia' AND SM. Location_name = 'Jarvselja' AND SM. Sample_pk = RSM. Sample_pk AND RSM. Sequence_pk = RS. Sequence_pk AND RS. RefSeq NOT IN (SELECT RS2. RefSeq FROM SampleMetadata1 SM2, RefSampleSequence RS2, RefSeqSampleMapping RSM2 WHERE SM2. Country_name = 'Estonia' AND SM2. Location_name = 'Satakunta' AND SM2. Sample_pk = RSM2. Sample_pk AND RSM2. Sequence_pk = RS2. Sequence_pk; 5075 tuples

\section{Observance of a specific fungal class}

Query 3 (Q3): provide a list of locations (i.e. countries) where particular ZOTUs belonging to a specific class (i.e. Fungal family) are detected.

SELECT DISTINCT RS. Sequence_pk, SM. Country_name, URS.GenusName FROM SampleMetadata1 SM, RefSampleSequence RS, RefSeqSampleMapping RSM, UniteRefSequence URS, AssignTaxa AT WHERE URS.GenusName = 'Lactarius' AND URS. RefSH_pk = AT. RefSH_pk AND $A T$. Sequence_pk = RS. Sequence_pk AND RS. Sequence_pk $=$ RSM. Sequence_pk AND RSM. Sample_pk = SM. Sample_pk; 339 tuples

\section{Appendix C: MonetDB queries used for genereating the outputs shown in Section 5 Results}

\section{Fungal diversity query}

Example 1: Retrieve ZOTUs for each phylum for a continent

SELECT DISTINCT RTDB.Phylum_name as phylum, count(DISTINCT

AT.Refsequence_pk) AS zotus

FROM Sample as S, Contain as C, RefSequence as RS, AssignTaxa as AT, RefTaxonomicDB as RTDB

WHERE S. country_continent $=$ 'Europe’ AND S.Sample_pk $=$ C. Sample_pk AND

C. Refsequence_pk = RS. Refsequence_pk AND RS. Refsequence_pk =

AT. Refsequence_pk AND AT. percentage_similarity $>70$ AND

AT. Refsequence_taxonomic_pk = RTDB.Refsequence_taxonomic_pk

GROUP BY RTDB.Phylum_name

\section{Fungal distribution query}

Example 2: Select a specific family and provide the ZOTU representatives of the family within the spatial range between the Tropic of cancer and Tropic of Capricorn

SELECT DISTINCT S.Sample_pk AS plot_id, S.sample_location_country AS

country, S.location_split2 AS location, ABS(S.sample_latitude) AS

dist_from_equator, count(C.Refsequence_pk) as fam_div, count(distinct 
RTDB.sh_unite_id) as unite_div

FROM SampleMD as S, Contain as C, RefSequence as RS, AssignTaxa as AT, RefTaxonomicDB as RTDB

WHERE RTDB.kingdom_name LIKE 'Fungi\%' AND RTDB. family_name LIKE

'Russulaceae\%' AND RTDB.Refsequence_taxonomic_pk =

AT.Refsequence_taxonomic_pk AND AT. Refsequence_pk = RS. Refsequence_pk AND

RS.Refsequence_pk $=$ C.Refsequence_pk AND C.Sample_pk $=$ S.Sample_pk AND ABS(S.sample_latitude) $<=$

23.5

GROUP BY (S.Sample_pk), S.sample_location_country, S.location_split2,

RTDB.family_name, S.sample_latitude

ORDER BY fam_div DESC

\section{Shared ZOTUs query}

\section{Example 3: Retrieving shared data among studies Query1 retrieves ZOTUs grouped for each continent. Query2 displays how to retrieve data shared among attributes, in this case ZOTUs among continents.}

SELECT DISTINCT S. country_continent AS continent, count(DISTINCT

S.country_geoname_pref_en) AS countries, count(DISTINCT S.Sample_pk) AS

samples, count(DISTINCT RS. RefSequence_pk) AS zotus

FROM SampleMD S, Contain C, RefSequence RS

WHERE S.Sample_pk = C. Sample_pk AND C. RefSequence_pk = RS.RefSequence_pk

GROUP BY S. country_continent

SELECT DISTINCT S. country_continent AS continent, count (DISTINCT

RS.RefSequence_pk) AS zotus FROM SampleMD S, Contain C, RefSequence RS

WHERE S.Sample_pk = C. Sample_pk AND C. RefSequence_pk = RS. RefSequence_pk

AND RS. RefSequence_pk IN (SELECT RS2. RefSequence_pk AS zotus2

FROM SampleMD S2, Contain C2, RefSequence RS2

WHERE S2. country_continent $=$ 'South America' AND S2. Sample_pk =

C2. Sample_pk AND C2. RefSequence_pk = RS2. RefSequence_pk)

GROUP BY S. country_continent

\section{References}

1. Zhang J, Elser JJ. Carbon:Nitrogen:Phosphorus Stoichiometry in Fungi: a Meta-Analysis. Front Microbiol 2017;8:1281. https:// doi.org/10.3389/fmicb.2017.01281.

2. Lange L. The importance of fungi and mycology for addressing major global challenges. IMA Fungus 2014;5:463-71. https:// doi.org/10.5598/imafungus.2014.05.02.10.

3. Treseder KK, Lennon JT. Fungal traits that drive ecosystem dynamics on land. Microbiol Mol Biol Rev 2015;79:243-62. https:// doi.org/10.1128/mmbr.00001-15.

4. Sam QH, Chang MW, Chai LYA. The Fungal Mycobiome and Its Interaction with Gut Bacteria in the Host. Int J Mol Sci 2017;18. https://doi.org/10.3390/ijms18020330.

5. Powell JR, Rillig MC. Biodiversity of arbuscular mycorrhizal fungi and ecosystem function. New Phytol 2018;220:1059-75. https://doi.org/10.1111/nph.15119.

6. Faeth SH, Fagan WF. Fungal endophytes: common host plant symbionts but uncommon mutualists. Integr Comp Biol 2002;42: 360-8. https://doi.org/10.1093/icb/42.2.360.

7. Suh SO, McHugh JV, Pollock DD, Blackwell M. The beetle gut: a hyperdiverse source of novel yeasts. Mycol Res 2005;109:2615. https://doi.org/10.1017/s0953756205002388.

8. Blackwell M. The fungi: 1, 2, 3 ... 5.1 million species? Am J Bot 2011;98:426-38. https://doi.org/10.3732/ajb.1000298. 
9. Shang Y, Feng P, Wang C. Fungi That Infect Insects: Altering Host Behavior and Beyond. PLoS Pathog 2015;11:e1005037. https://doi.org/10.1371/journal.ppat.1005037.

10. Crowther TW, Boddy L, Hefin Jones T. Functional and ecological consequences of saprotrophic fungus-grazer interactions. ISME J 2012;6:1992-2001. https://doi.org/10.1038/ismej.2012.53.

11. Hawksworth DL, Lücking R. Fungal Diversity Revisited: 2.2 to 3.8 Million Species. Microbiol Spectr 2017;5. https://doi.org/10. 1128/9781555819583.ch4.

12. Willis KJ. State of the World's Fungi 2018. Report. Royal Botanic Gardens, Kew. Available from: https://stateoftheworldsfungi. org/2018/reports/SOTWFungi_2018_Full_Report.pdf.

13. Hawksworth DL. The fungal dimension of biodiversity: magnitude, significance, and conservation. Mycol Res 1991;95:641-55. https://doi.org/10.1016/s0953-7562(09)80810-1.

14. Lindahl BD, Henrik Nilsson R, Tedersoo L, Abarenkov K, Carlsen T, Kjøller R, et al. Fungal community analysis by high-throughput sequencing of amplified markers - a user's guide. New Phytol 2013;199:288-99. https://doi.org/10.1111/nph.12243.

15. Hibbett D, Abarenkov K, Kõljalg U, Öpik M, Chai B, Cole J, et al. Sequence-based classification and identification of Fungi. Mycologia 2016;108:1049-68. https://doi.org/10.3852/16-130.

16. Waksman SA. A Method for Counting the Number of Fungi in the Soil. J Bacteriol 1922;7:339-41. https://doi.org/10.1128/jb.7. 3.339-341.1922.

17. Kirk JL, Beaudette LA, Hart M, Moutoglis P, Klironomos JN, Lee H, et al. Methods of studying soil microbial diversity. J Microbiol Methods 2004;58:169-88. https://doi.org/10.1016/j.mimet.2004.04.006.

18. Shumway M, Cochrane G, Sugawara H. Archiving next generation sequencing data. Nucleic Acids Res 2010;38:D870-1. https://doi.org/10.1093/nar/gkp1078.

19. Peay KG, Kennedy PG, Bruns TD. Fungal community ecology: a hybrid beast with a molecular master. Bioscience 2008;58: 799-810. https://doi.org/10.1641/b580907.

20. Straatsma G, Ayer F, Egli S. Species richness, abundance, and phenology of fungal fruit bodies over 21 years in a Swiss forest plot. Mycological Research 2001;105:515-23. https://doi.org/10.1017/s0953756201004154.

21. Taylor DL, Herriott IC, Stone KE, McFarland JW, Booth MG, Leigh MB. Structure and resilience of fungal communities in Alaskan boreal forest soils. Can J For Res 2010;40:1288-301. https://doi.org/10.1139/x10-081.

22. Gardes M, Bruns TD. Community structure of ectomycorrhizal fungi in a Pinus muricata forest: above- and below-ground views. Can J Bot 1996;74:1572-83. https://doi.org/10.1139/b96-190.

23. Begerow D, Nilsson H, Unterseher M, Maier W. Current state and perspectives of fungal DNA barcoding and rapid identification procedures. Appl Microbiol Biotechnol 2010;87:99-108. https://doi.org/10.1007/s00253-010-2585-4.

24. Ratnasingham S, Hebert PDN. bold: The Barcode of Life Data System (http://www.barcodinglife.org). Mol Ecol Notes 2007;7: 355-64. https://doi.org/10.1111/j.1471-8286.2007.01678.x.

25. O’Brien HE, Parrent JL, Jackson JA, Moncalvo JM, Vilgalys R. Fungal community analysis by large-scale sequencing of environmental samples. Appl Environ Microbiol 2005;71:5544-50. https://doi.org/10.1128/aem.71.9.5544-5550.2005.

26. Schoch CL, Seifert KA, Huhndorf S, Robert V, Spouge JL, Levesque CA, et al. Nuclear ribosomal internal transcribed spacer (ITS) region as a universal DNA barcode marker for Fungi. Proc Natl Acad Sci USA 2012;109:6241-46. https://doi.org/10.1073/ pnas.1117018109.

27. Quince C, Lanzén A, Curtis TP, Davenport RJ, Hall N, Head IM, et al. Accurate determination of microbial diversity from 454 pyrosequencing data. Nat Methods 2009;6:639-41. https://doi.org/10.1038/nmeth.1361.

28. Kemler M, Garnas J, Wingfield MJ, Gryzenhout M, Pillay KA, Slippers B. Ion Torrent PGM as Tool for Fungal Community Analysis: A Case Study of Endophytes in Eucalyptus grandis Reveals High Taxonomic Diversity. PLoS One 2013;8:e81718. https://doi. org/10.1371/journal.pone.0081718.

29. Bálint M, Schmidt PA, Sharma R, Thines M, Schmitt I. An Illumina metabarcoding pipeline for fungi. Ecol Evol 2014;4:2642-53. https://doi.org/10.1002/ece3.1107.

30. Zimmerman NB, Vitousek PM. Fungal endophyte communities reflect environmental structuring across a Hawaiian landscape. Proc Natl Acad Sci USA 2012;109:13022-7. https://doi.org/10.1073/pnas.1209872109.

31. Taylor DL, Hollingsworth TN, McFarland JW, Lennon NJ, Nusbaum C, Ruess RW. A first comprehensive census of fungi in soil reveals both hyperdiversity and fine-scale niche partitioning. Ecol Monogr 2014;84:3-20. https://doi.org/10.1890/12-1693.1.

32. Op De Beeck M, Lievens B, Busschaert P, Declerck S, Vangronsveld J, Colpaert JV. Comparison and validation of some ITS primer pairs useful for fungal metabarcoding studies. PLoS One 2014;9:e97629. https://doi.org/10.1371/journal.pone. 0097629.

33. Geml J, Gravendeel B, van der Gaag KJ, Neilen M, Lammers Y, Raes N, et al. The contribution of DNA metabarcoding to fungal conservation: diversity assessment, habitat partitioning and mapping red-listed fungi in protected coastal Salix repens communities in the Netherlands. PLoS One 2014;9:e99852. https://doi.org/10.1371/journal.pone.0099852.

34. Zhang T, Wang NF, Zhang YQ, Liu HY, Yu LY. Diversity and distribution of fungal communities in the marine sediments of Kongsfjorden, Svalbard (High Arctic). Sci Rep 2015;5:14524. https://doi.org/10.1038/srep14524.

35. Roy-Bolduc A, Laliberté E, Boudreau S, Hijri M. Strong linkage between plant and soil fungal communities along a successional coastal dune system. FEMS Microbiol Ecol 2016 Oct;92. https://doi.org/10.1093/femsec/fiw156. 
36. Tedersoo L, Bahram M, Põlme S, Kõljalg U, Yorou NS, Wijesundera R, et al. Fungal biogeography. Global diversity and geography of soil fungi. Science 2014;346:1256688. https://doi.org/10.1126/science.1256688.

37. Davison J, Moora M, Öpik M, Adholeya A, Ainsaar L, Bâ A, et al. Global assessment of arbuscular mycorrhizal fungus diversity reveals very low endemism. Science 2015;349:970-3. https://doi.org/10.1126/science.aab1161.

38. Leff JW, Jones SE, Prober SM, Barberán A, Borer ET, Firn JL, et al. Consistent responses of soil microbial communities to elevated nutrient inputs in grasslands across the globe. Proc Natl Acad Sci USA 2015;112:10967-72. https://doi.org/10.1073/ pnas.1508382112.

39. Adams RI, Miletto M, Taylor JW, Bruns TD. Dispersal in microbes: fungi in indoor air are dominated by outdoor air and show dispersal limitation at short distances. ISME J 2013;7:1262-73. https://doi.org/10.1038/ismej.2013.28.

40. Brown SP, Callaham MA, Jr, Oliver AK, Jumpponen A. Deep Ion Torrent sequencing identifies soil fungal community shifts after frequent prescribed fires in a southeastern US forest ecosystem. FEMS Microbiol Ecol 2013;86:557-66. https://doi.org/10. 1111/1574-6941.12181.

41. Jarvis SG, Woodward S, Taylor AFS. Strong altitudinal partitioning in the distributions of ectomycorrhizal fungi along a short (300 m) elevation gradient. New Phytol 2015;206:1145-55. https://doi.org/10.1111/nph.13315.

42. Peay KG, Russo SE, McGuire KL, Lim Z, Chan JP, Tan S, et al. Lack of host specificity leads to independent assortment of dipterocarps and ectomycorrhizal fungi across a soil fertility gradient. Ecol Lett 2015;18:807-16. https://doi.org/10.1111/ele. 12459.

43. Gomes SIF, Aguirre-Gutiérrez J, Bidartondo MI, Merckx VSFT. Arbuscular mycorrhizal interactions of mycoheterotrophic Thismia are more specialized than in autotrophic plants. New Phytol 2017;213:1418-27. https://doi.org/10.1111/nph. 14249.

44. Chen L, Zheng Y, Gao C, Mi XC, Ma KP, Wubet T, et al. Phylogenetic relatedness explains highly interconnected and nested symbiotic networks of woody plants and arbuscular mycorrhizal fungi in a Chinese subtropical forest. Mol Ecol 2017;26: 2563-75. https://doi.org/10.1111/mec.14061.

45. Gomes SIF, Merckx VSFT, Saavedra S. Fungal-host diversity among mycoheterotrophic plants increases proportionally to their fungal-host overlap. Ecol Evol 2017;7:3623-30. https://doi.org/10.1002/ece3.2974.

46. Peay KG, von Sperber C, Cardarelli E, Toju H, Francis CA, Chadwick OA, et al. Convergence and contrast in the community structure of Bacteria, Fungi and Archaea along a tropical elevation-climate gradient. FEMS Microbiol Ecol 2017;93. https:// doi.org/10.1093/femsec/fix045.

47. Cochrane G, Karsch-Mizrachi I, Takagi T, International Nucleotide Sequence Database Collaboration. The international nucleotide sequence database collaboration. Nucleic Acids Res 2015;44:D48-50. https://doi.org/10.1093/nar/gkv1323.

48. Cochrane G, Akhtar R, Bonfield J, Bower L, Demiralp F, Faruque N, et al. Petabyte-scale innovations at the European Nucleotide Archive. Nucleic Acids Res 2009;37(Database issue):D19-25. https://doi.org/10.1093/nar/gkn765.

49. Sugawara H, Ikeo K, Fukuchi S, Gojobori T, Tateno Y. DDBJ dealing with mass data produced by the second generation sequencer. Nucleic Acids Res 2009;37(Database issue):D16-8. https://doi.org/10.1093/nar/gkn724.

50. NCBI Documentation, SRA database growth. Available from: https://www.ncbi.nlm.nih.gov/sra/docs/sragrowth/.

51. Meyer F, Paarmann D, D'Souza M, Olson R, Glass EM, Kubal M, et al. The metagenomics RAST server - a public resource for the automatic phylogenetic and functional analysis of metagenomes. BMC Bioinformatics 2008;9:386. https://doi.org/10.1186/ 1471-2105-9-386.

52. Schloss PD, Westcott SL, Ryabin T, Hall JR, Hartmann M, Hollister EB, et al. Introducing mothur: open-source, platformindependent, community-supported software for describing and comparing microbial communities. Appl Environ Microbiol 2009;75:7537-41. https://doi.org/10.1128/aem.01541-09.

53. Caporaso JG, Kuczynski J, Stombaugh J, Bittinger K, Bushman FD, Costello EK, et al. QIIME allows analysis of high-throughput community sequencing data. Nat Methods 2010;7:335-6. https://doi.org/10.1038/nmeth.f.303.

54. Edgar RC. Search and clustering orders of magnitude faster than BLAST. Bioinformatics 2010;26:2460-1. https://doi.org/10. 1093/bioinformatics/btq461.

55. Hildebrand F, Tadeo R, Voigt AY, Bork P, Raes J. LotuS: an efficient and user-friendly OTU processing pipeline. Microbiome 2014;2:30. https://doi.org/10.1186/2049-2618-2-30.

56. Fosso B, Santamaria M, Marzano M, Alonso-Alemany D, Valiente G, Donvito G, et al. BioMaS: a modular pipeline for Bioinformatic analysis of Metagenomic AmpliconS. BMC Bioinformatics 2015;16:203. https://doi.org/10.1186/s12859-0150595-z.

57. Mysara M, Njima M, Leys N, Raes J, Monsieurs P. From reads to operational taxonomic units: an ensemble processing pipeline for MiSeq amplicon sequencing data. Gigascience 2017;6:1-10. https://doi.org/10.1093/gigascience/giw017.

58. Balzer S, Malde K, Jonassen I. Systematic exploration of error sources in pyrosequencing flowgram data. Bioinformatics 2011; 27:i304-9. https://doi.org/10.1093/bioinformatics/btr251.

59. Bakker MG, Tu ZJ, Bradeen JM, Kinkel LL. Implications of Pyrosequencing Error Correction for Biological Data Interpretation. PLoS One 2012;7:1-9. https://doi.org/10.1371/journal.pone.0044357.

60. Mysara M, Leys N, Raes J, Monsieurs P. NoDe: a fast error-correction algorithm for pyrosequencing amplicon reads. BMC Bioinformatics 2015;16:88. https://doi.org/10.1186/s12859-015-0520-5. 
61. Schirmer M, ljaz UZ, D’Amore R, Hall N, Sloan WT, Quince C. Insight into biases and sequencing errors for amplicon sequencing with the Illumina MiSeq platform. Nucleic Acids Res 2015;43:e37. https://doi.org/10.1093/nar/gku1341.

62. Schloss PD, Gevers D, Westcott SL. Reducing the Effects of PCR Amplification and Sequencing Artifacts on 16S rRNA-Based Studies. PLoS One 2011;6:1-14. https://doi.org/10.1371/journal.pone.0027310.

63. Kozich JJ, Westcott SL, Baxter NT, Highlander SK, Schloss PD. Development of a dual-index sequencing strategy and curation pipeline for analyzing amplicon sequence data on the MiSeq Illumina sequencing platform. Appl Environ Microbiol 2013;79: 5112-20. https://doi.org/10.1128/aem.01043-13.

64. Edgar RC, Flyvbjerg H. Error filtering, pair assembly and error correction for next-generation sequencing reads. Bioinformatics 2015;31:3476-82. https://doi.org/10.1093/bioinformatics/btv401.

65. Altschul SF, Madden TL, Schäffer AA, Zhang J, Zhang Z, Miller W, et al. Gapped BLAST and PSI-BLAST: a new generation of protein database search programs. Nucleic Acids Res 1997;25:3389-402. https://doi.org/10.1093/nar/25.17.3389.

66. Kõljalg U, Nilsson RH, Abarenkov K, Tedersoo L, Taylor AFS, Bahram M, et al. Towards a unified paradigm for sequence-based identification of fungi. Mol Ecol 2013;22:5271-7. https://doi.org/10.1111/mec.12481.

67. Nilsson RH, Anslan S, Bahram M, Wurzbacher C, Baldrian P, Tedersoo L. Mycobiome diversity: high-throughput sequencing and identification of fungi. Nat Rev Microbiol 2019;17:95-109. https://doi.org/10.1038/s41579-018-0116-y.

68. Nilsson RH, Larsson KH, Taylor AFS, Bengtsson-Palme J, Jeppesen TS, Schigel D, et al. The UNITE database for molecular identification of fungi: handling dark taxa and parallel taxonomic classifications. Nucleic Acids Res 2019;47:D259-64. https://doi.org/10.1093/nar/gky1022.

69. Looney BP, Ryberg M, Hampe F, Sánchez-García M, Matheny PB. Into and out of the tropics: global diversification patterns in a hyperdiverse clade of ectomycorrhizal fungi. Mol Ecol 2016;25:630-47. https://doi.org/10.1111/mec.13506.

70. Henrik Nilsson R, Tedersoo L, Lindahl BD, Kjøller R, Carlsen T, Quince C, et al. Towards standardization of the description and publication of next-generation sequencing datasets of fungal communities. New Phytol 2011;191:314-8. https://doi.org/10. 1111/j.1469-8137.2011.03755.x.

71. Meiser A, Bálint M, Schmitt I. Meta-analysis of deep-sequenced fungal communities indicates limited taxon sharing between studies and the presence of biogeographic patterns. New Phytol 2014;201:623-35. https://doi.org/10.1111/nph. 12532.

72. Öpik M, Vanatoa A, Vanatoa E, Moora M, Davison J, Kalwij JM, et al. The online database MaarjAM reveals global and ecosystemic distribution patterns in arbuscular mycorrhizal fungi (Glomeromycota). New Phytol 2010;188:223-41. https:// doi.org/10.1111/j.1469-8137.2010.03334.x.

73. Benson D, Cavanaugh M, Clark K, Karsch-Mizrachi I, Lipman D, Ostell J, et al. GenBank. Nucleic Acids Res.2013;41(Database issue):D36-42. https://doi.org/10.1093/nar/gks1195.

74. Bruns TD, Taylor JW. Comment on "Global assessment of arbuscular mycorrhizal fungus diversity reveals very low endemism". Science 2016;351:826. https://doi.org/10.1126/science.aad4228.

75. PubMed: The Bibliographic Database. The National Center for Biotechnology Information Handbook. 2nd ed.; 2013. 2002 Oct 9 [Updated 2013 Mar 20]. Available from: https://www.ncbi.nlm.nih.gov/books/NBK153385/.

76. PMC-ID Converter API. National Center for Biotechnology Information (US). Available from: https://www.ncbi.nlm.nih.gov/ pmc/tools/id-converter-api/.

77. Biopython Module Parser. Available from: https://biopython.org/DIST/docs/api/Bio.Entrez.Parser-module.html.

78. Entrez Programming Utilities (E-utilities) Help. National Center for Biotechnology Information (US); 2010. Available from: https://www.ncbi.nlm.nih.gov/books/NBK25501/.

79. Barrett T, Clark K, Gevorgyan R, Gorelenkov V, Gribov E, Karsch-Mizrachi I, et al. BioProject and BioSample databases at NCBI: facilitating capture and organization of metadata. Nucleic Acids Res 2012;40(Database issue):D57-63. https://doi.org/10. 1093/nar/gkr1163.

80. MEDLINE®PubMed $\circledast$ XML Element Descriptions and their Attributes. U.S. National Library of Medicine; 2005. Available from: https://www.nlm.nih.gov/bsd/licensee/elements_descriptions.html.

81. PyPDF2: Pure-Python library built as a PDF toolkit. Available from: https://pypi.org/project/PyPDF2/.

82. Download Guide. SRA Handbook. National Center for Biotechnology Information (US); 2016. Available from: https://www. ncbi.nlm.nih.gov/books/NBK242621/.

83. SRA Toolkit. SRA Toolkit Development Team, National Center for Biotechnology Information (US); 2011. Available from: http:// ncbi.github.io/sra-tools.

84. Yilmaz P, Kottmann R, Field D, Knight R, Cole JR, Amaral-Zettler L, et al. The "Minimum Information about an ENvironmental Sequence" (MIENS) specification. Nature Precedings 2010. https://doi.org/10.1038/npre.2010.5252.1.

85. Yilmaz P, Kottmann R, Field D, Knight R, Cole JR, Amaral-Zettler L, et al. Minimum information about a marker gene sequence (MIMARKS) and minimum information about any (x) sequence (MIxS) specifications. Nat Biotechnol 2011 May;29:415-20. https://doi.org/10.1038/nbt.1823.

86. Wilkinson MD, Dumontier M, Aalbersberg IJ, Appleton G, Axton M, Baak A, et al. The FAIR Guiding Principles for scientific data management and stewardship. Sci Data 2016 Mar;3:160018. https://doi.org/10.1038/sdata.2016.18. 
87. Glass EM, Dribinsky Y, Yilmaz P, Levin H, Van Pelt R, Wendel D, et al. MIxS-BE: a MIxS extension defining a minimum information standard for sequence data from the built environment. ISME J 2014 Jan;8:1-3. https://doi.org/10.1038/ismej. 2013.176.

88. Robertson T, Döring M, Guralnick R, Bloom D, Wieczorek J, Braak K, et al. The GBIF integrated publishing toolkit: facilitating the efficient publishing of biodiversity data on the internet. PLoS One 2014 Aug;9:e102623. https://doi.org/10.1371/journal. pone.0102623.

89. Penev L, Mietchen D, Chavan V, Hagedorn G, Smith V, Shotton D, et al. Strategies and guidelines for scholarly publishing of biodiversity data. Research Ideas and Outcomes 2017;3:e12431. https://doi.org/10.3897/rio.3.e12431.

90. Gross T, Taylor AG, Joudrey DN. Still a Lot to Lose: The Role of Controlled Vocabulary in Keyword Searching. Catal Clas Quart 2014;53:1-39. https://doi.org/10.1080/01639374.2014.917447.

91. Bernstein MN, Doan A, Dewey CN. MetaSRA: normalized human sample-specific metadata for the Sequence Read Archive. Bioinformatics 2017 Sep;33:2914-23. https://doi.org/10.1093/bioinformatics/btx334.

92. Python Geocoder. Simple and consistent geocoding library written in Python. Available from: https://pypi.org/project/ geocoder/.

93. GeoNames. The GeoNames geographical database. Available from: http://www.geonames.org.

94. Buttigieg PL, Morrison N, Smith B, Mungall CJ, Lewis SE, ENVO Consortium. The environment ontology: contextualising biological and biomedical entities. J Biomed Semantics 2013 Dec;4:43. https://doi.org/10.1186/2041-1480-4-43.

95. Pafilis E, Bērziñš R, Jensen LJ. EXTRACT 2.0: text-mining-assisted interactive annotation of biomedical named entities and ontology terms. Biodiv Inform Sci Stand 2017;1:e20152. https://doi.org/10.1101/111088.

96. Stackebrandt E, Goebel BM. Taxonomic Note: A Place for DNA-DNA Reassociation and 16S rRNA Sequence Analysis in the Present Species Definition in Bacteriology. Int J Syst Evol Microbiol 1994;44:846-9. https://doi.org/10.1099/00207713-444-846.

97. Edgar RC. UNOISE2: improved error-correction for Illumina $16 \mathrm{~S}$ and ITS amplicon sequencing. bioRxiv 2016. https://doi.org/ $10.1101 / 081257$.

98. Rideout JR, He Y, Navas-Molina JA, Walters WA, Ursell LK, Gibbons SM, et al. Subsampled open-reference clustering creates consistent, comprehensive OTU definitions and scales to billions of sequences. Peer) 2014;2:e545. https://doi.org/10.7717/ peerj.545.

99. Callahan BJ, McMurdie PJ, Holmes SP. Exact sequence variants should replace operational taxonomic units in marker-gene data analysis. ISME J 2017 Dec;11:2639-43. https://doi.org/10.1038/ismej.2017.119.

100. Edgar RC. Updating the $97 \%$ identity threshold for 16 S ribosomal RNA OTUs. Bioinformatics 2018 Jul;34:2371-5. https://doi. org/10.1093/bioinformatics/bty113.

101. Edgar RC. UPARSE: highly accurate OTU sequences from microbial amplicon reads. Nat Methods 2013 Oct;10:996-8. https:// doi.org/10.1038/nmeth.2604.

102. Ferreira JE, Takai OK. Understanding Database Design. In: Bioinformatics in tropical disease research: A aractical and casestudy approach. National Center for Biotechnology Information (US); 2007. Available from: https://www.ncbi.nlm.nih.gov/ books/NBK6828/.

103. MonetDB: Open source column-oriented database management system;. Available from: https://www.monetdb.org.

104. Idreos S, Groffen F, Nes N, Manegold S, Sjoerd Mullender K, Kersten ML. MonetDB: Two decades of research in columnoriented database architectures. IEEE Data Eng Bull 2012;35. http://sites.computer.org/debull/A12mar/monetdb.pdf.

105. Boncz PA, Kersten ML, Manegold S. Breaking the memory wall in MonetDB. Communications of the ACM 2008;51:77-85. https://doi.org/10.1145/1409360.1409380.

106. Ivanova M, Nes N, Goncalves R, Kersten M. MonetDB/SQL Meets SkyServer: the Challenges of a Scientific Database. In: 19th International Conference on Scientific and Statistical Database Management (SSDBM 2007). IEEE; 2007. p. 13. https://doi. org/10.1109/SSDBM.2007.19.

107. Liarou E, Idreos S, Manegold S, Kersten M. MonetDB/DataCell. Proc VLDB Endowment 2012;5:1910-3. https://doi.org/10. $14778 / 2367502.2367535$.

108. Vermeij M, Quak W, Kersten M, Nes N. Monetdb, a novel spatial columnstore dbms. In: Academic Proceedings of the 2008 Free and Open Source for Geospatial (FOSS4G) Conference, OSGeo; 2008. p. 193-9. https://citeseerx.ist.psu.edu/viewdoc/ summary?doi=10.1.1.464.7219.

109. GeoSpatial - MonetDB. Available from: https://www.monetdb.org/Documentation/Extensions/GIS.

110. Cijvat R, Manegold S, Kersten M, Klau GW, Schönhuth A, Marschall T, et al. Genome sequence analysis with MonetDB. Datenbank-Spektrum 2015;15:185-91. https://doi.org/10.1007/s13222-015-0198-x.

111. Martinez-Rubi O, van Oosterom P, Gonçalves R, Tijssen T, Ivanova M, Kersten ML, et al. Benchmarking and improving point cloud data management in MonetDB. SIGSPATIAL Special 2015 Mar;6:11-8. https://doi.org/10.1145/2744700.2744702.

112. Liarou E, Goncalves R, Idreos S. Exploiting the power of relational databases for efficient stream processing. In: Proceedings of the 12th International Conference on Extending Database Technology Advances in Database Technology - EDBT '09. New York, NY, USA: ACM Press; 2009. 323-34. https://doi.org/10.1145/1516360.1516398. 
113. Abadi D, Madden S, Ferreira M. Integrating compression and execution in column-oriented database systems. In: Proceedings of the 2006 ACM SIGMOD International Conference on Management of Data - SIGMOD '06. New York, NY, USA: Association for Computing Machinery; 2006. 671-82. https://doi.org/10.1145/1142473.1142548.

114. Primer Sequences - the Bruns Lab, Useful Items. Available from: https://nature.berkeley.edu/brunslab/tour/primers.html.

115. PDFMiner - Python PDF parser and analyzer, pdfminer-docs 0.0.1 documentation. Available from: https://pdfminer-docs. readthedocs.io/pdfminer_index.html.

116. NCBITools Sequence Read Archieve Browsing. Available from: https://trace.ncbi.nlm.nih.gov/Traces/sra/sra.cgi? view=studies.

117. Zhu Y, Stephens RM, Meltzer PS, Davis SR. SRAdb: query and use public next-generation sequencing data from within R. BMC Bioinformatics 2013 Jan;14:19. https://doi.org/10.1186/1471-2105-14-19.

118. SRAMongo package - A package to download metadata from SRA/Biosample/Geo and dump into a mongo database. Available from: https://pypi.org/project/sramongo/.

119. Leinonen R, Sugawara H, Shumway M, International Nucleotide Sequence Database Collaboration. The sequence read archive. Nucleic Acids Res 2011 Jan;39(Database issue):D19-21. https://doi.org/10.1093/nar/gkq1019.

120. Sayers EW, Agarwala R, Bolton EE, Brister J, Canese K, Clark K, et al. Database resources of the National Center for Biotechnology Information. Nucleic Acids Res 2018;47:D23-8. https://doi.org/10.1093/nar/gky1069.

121. Degtyarenko K, de Matos P, Ennis M, Hastings J, Zbinden M, McNaught A, et al. ChEBI: a database and ontology for chemical entities of biological interest. Nucleic Acids Res 2008 Jan;36:D344-50. https://doi.org/10.1093/nar/gkm791.

122. Gkoutos GV, Schofield PN, Hoehndorf R. The Units Ontology: a tool for integrating units of measurement in science. Database 2012:bas033. https://doi.org/10.1093/database/bas033.

123. Mons B, Neylon C, Velterop J, Dumontier M, da Silva Santos LOB, Wilkinson MD. Cloudy, increasingly FAIR; revisiting the FAIR Data guiding principles for the European Open Science Cloud. ISU 2017 Mar;37:49-56. https://doi.org/10.3233/isu-170824. 